Supporting Information

\title{
Substrate Engineering in Lipase-catalyzed Selective Polymerization of $D$-/L-Aspartates and Diols to Prepare Helical Chiral Polyester
}

\author{
Yu Zhang, ${ }^{\dagger,}$ Bo Xia, ${ }^{*},+, \S$ Yanyan Li, ${ }^{\dagger}$ Xianfu Lin, ${ }^{\dagger}$ Qi Wu ${ }^{\dagger, *}$ \\ $\dagger$ Department of Chemistry, Zhejiang University, Hangzhou 310027, People’s Republic of China; \\ $\ddagger$ Jiyang College of Zhejiang A\&F University, Zhuji 311800, People’s Republic of China;
}




\section{Supplemental figures and tables in Supporting Information}

Table S1. $T_{5 \%}$ and $T_{d \max }$ of chiral polyesters poly $(L-1 a), \operatorname{poly}(L-3 a), \operatorname{poly}(L-2 a), \operatorname{poly}(L-9 a)$ and $\operatorname{poly}(D-9 a)$ a, b

\begin{tabular}{cccc}
\hline Polyester & $T_{5 \%}\left({ }^{\circ} \mathrm{C}\right)^{\mathrm{c}}$ & $T_{\text {dmax- }}\left({ }^{\circ} \mathrm{C}\right)$ & $T_{\text {dmax-2 }}\left({ }^{\circ} \mathrm{C}\right)$ \\
\hline Poly $(\boldsymbol{L}$-1a) & 295 & $-{ }^{\mathrm{d}}$ & 387 \\
Poly $(\boldsymbol{L}$-2a $)$ & 232 & 274 & 399 \\
Poly $(\boldsymbol{L}$-3a $)$ & 249 & 285 & 399 \\
Poly $(\boldsymbol{L}$-9a) & 231 & 251 & 399 \\
Poly $(\boldsymbol{D}$-9a) & 225 & 250 & 399 \\
\hline
\end{tabular}

${ }^{\text {a }} \mathrm{TG}$ was used for determing the thermal stability of chiral polyesters, $30-700{ }^{\circ} \mathrm{C}, 10^{\circ} \mathrm{C} / \mathrm{min} ;{ }^{\mathrm{b}} T_{d \max }$ refered to the temperatures of maximal rate of decomposition; ${ }^{\mathrm{c}} T_{5} \%$ refered to the temperatures at $5 \%$ weight loss; ${ }^{\mathrm{d}}$ "-" refered to not detect.

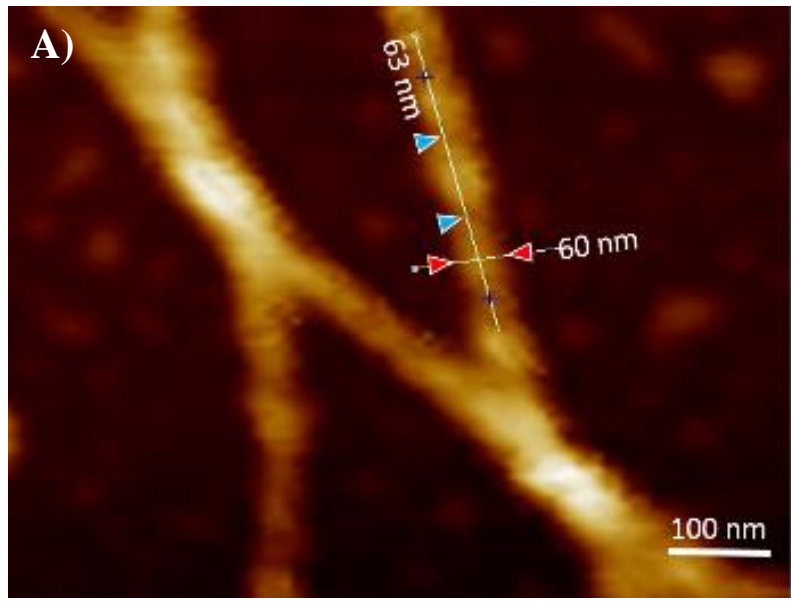

B)

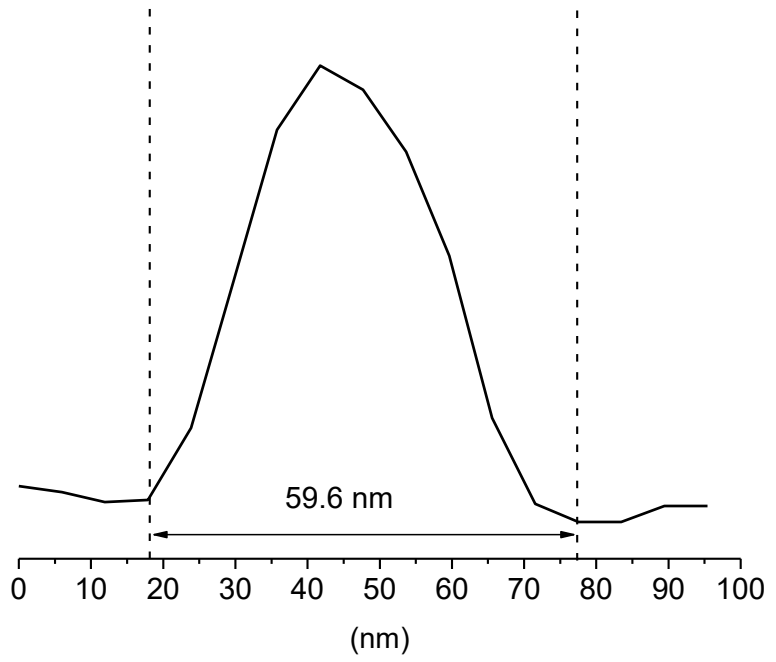

C)

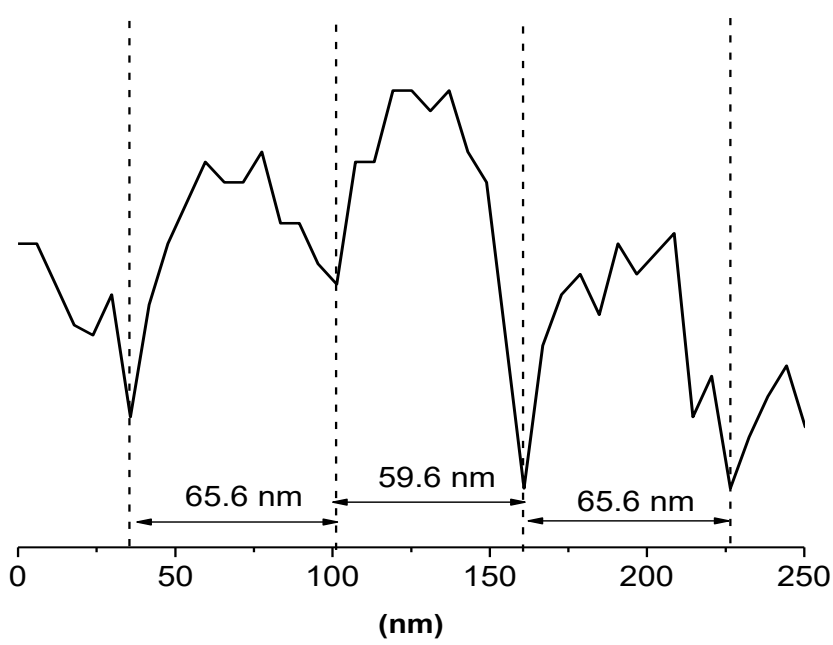

Figure S1. AFM analysis of poly(L-9a): A) AFM image of poly(L-9a); B) Helix diameter; C) Helix pitch 


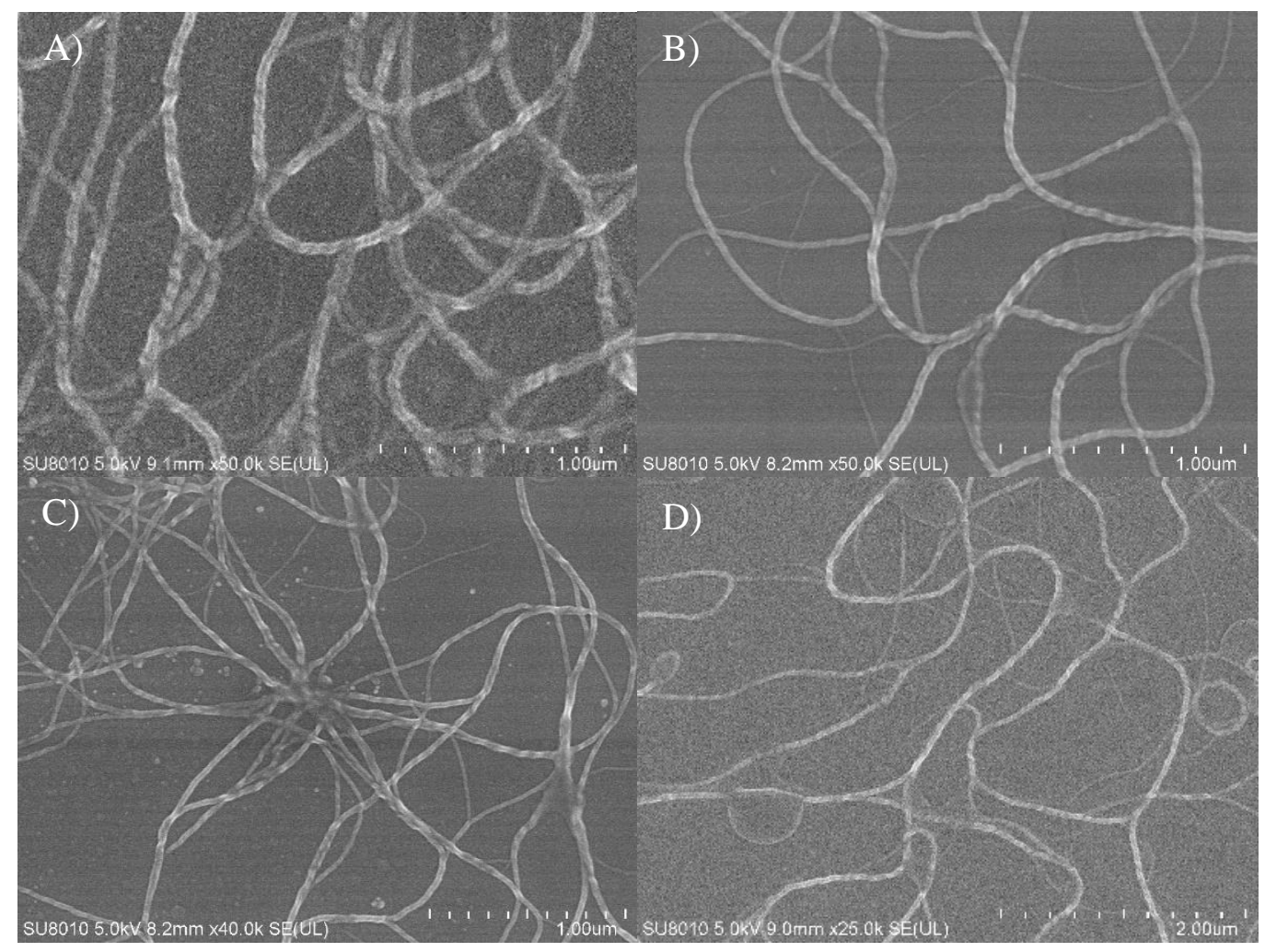

Figure S2. SEM images of chiral polyesters. A) Poly (L-1a); B) Poly (L-2a); C) Poly (L-3a); D) Poly (D-9a). The concentration of polymers solution in DCM was $0.5 \mathrm{mg} / \mathrm{mL}$, and the ratio of hexane/DCM (poor solvent/good solvent) used in the preparation procedure was 2:1. 


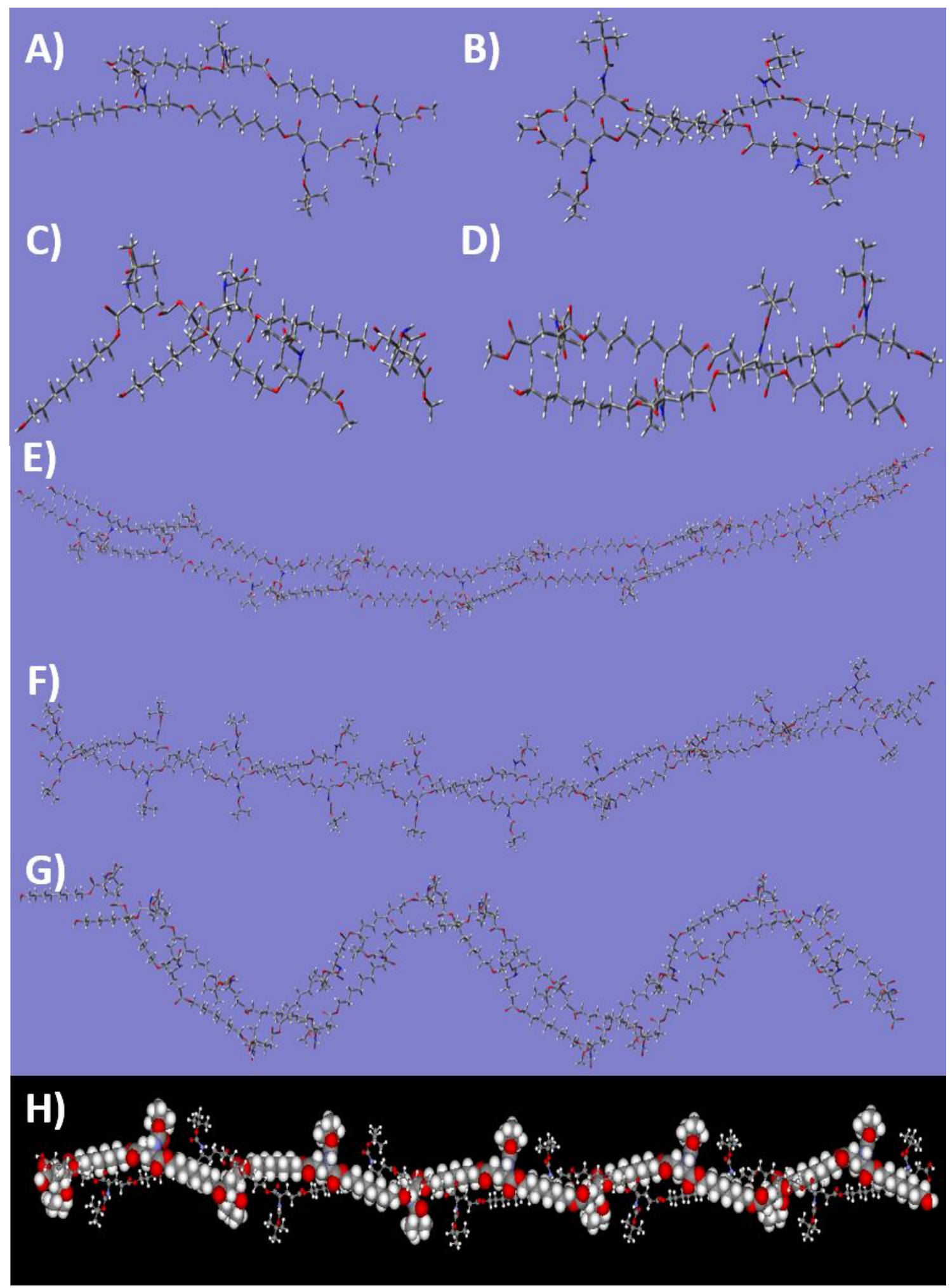

Figure S3. Geometry optimization of the two-chain system of Poly (L-9a): A)-D): dimer; E)-H): ten repeating units. 
2. ${ }^{1} \mathrm{H}$ NMR spectra of chiral polyesters

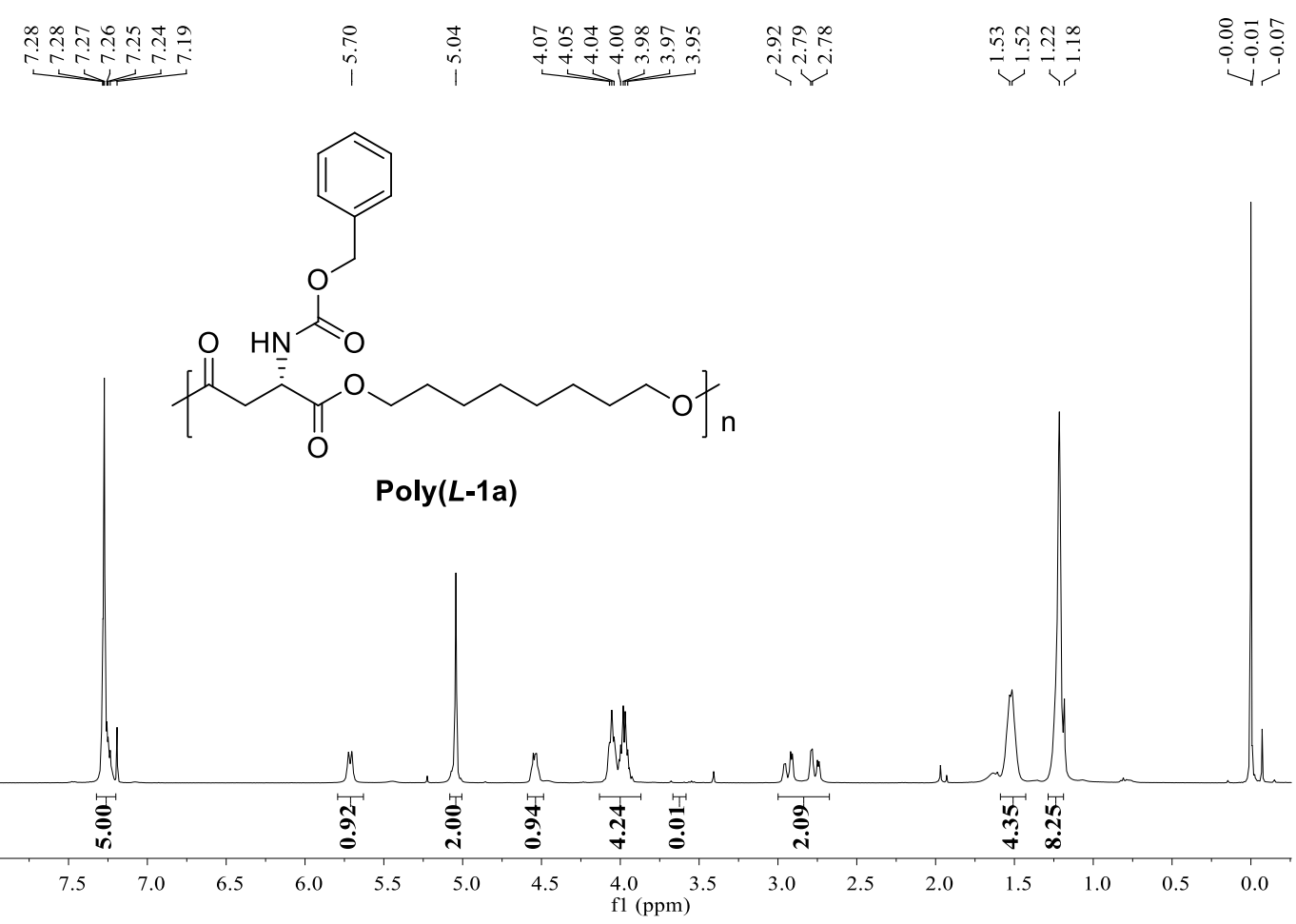

Figure S4. ${ }^{1} \mathrm{H}-\mathrm{NMR}$ spectrum of Poly $(\boldsymbol{L}-1 \mathrm{a})$

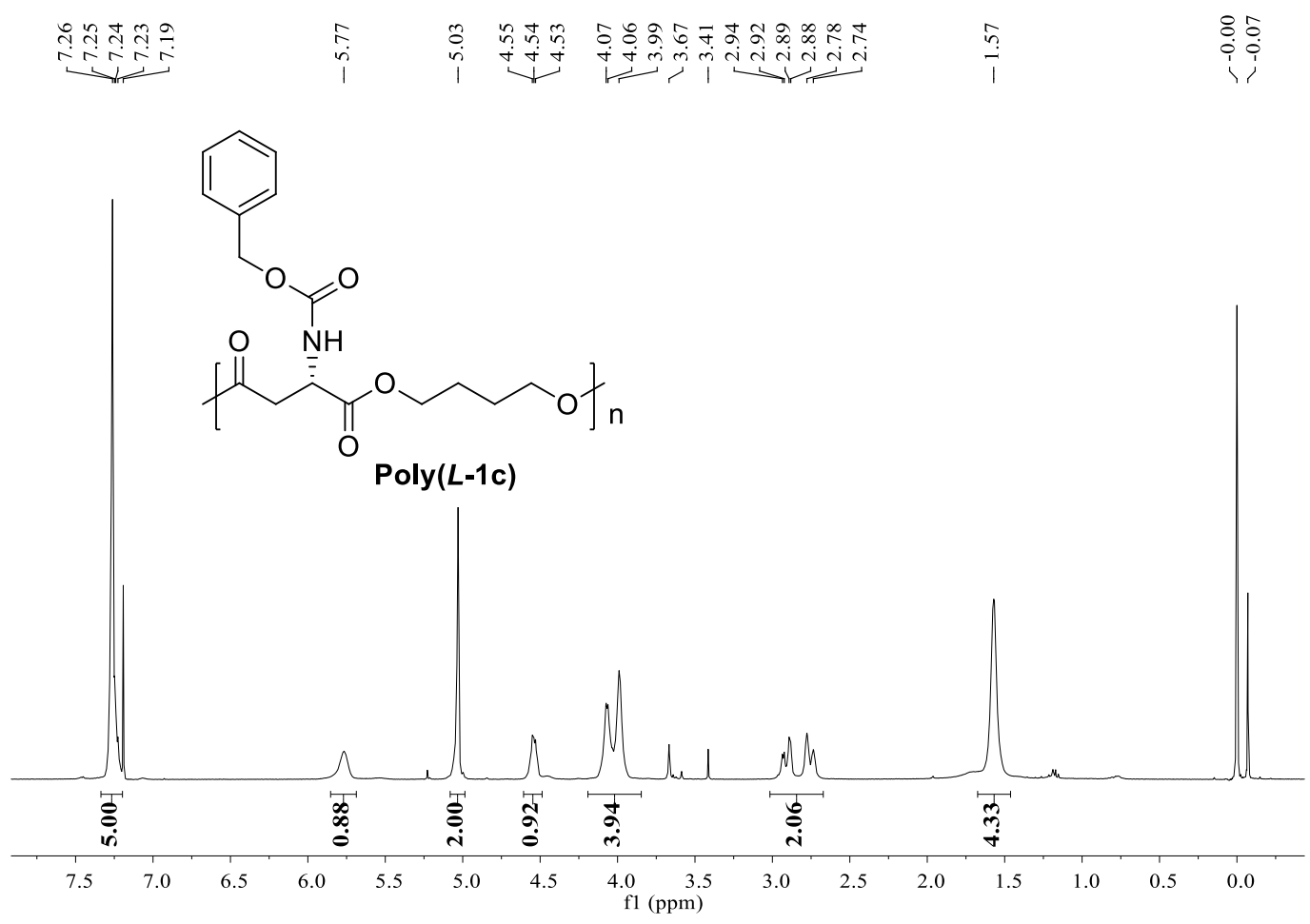

Figure S5. ${ }^{1} \mathrm{H}-\mathrm{NMR}$ pectrum of Poly $(\boldsymbol{L}-\mathbf{1 c})$ 


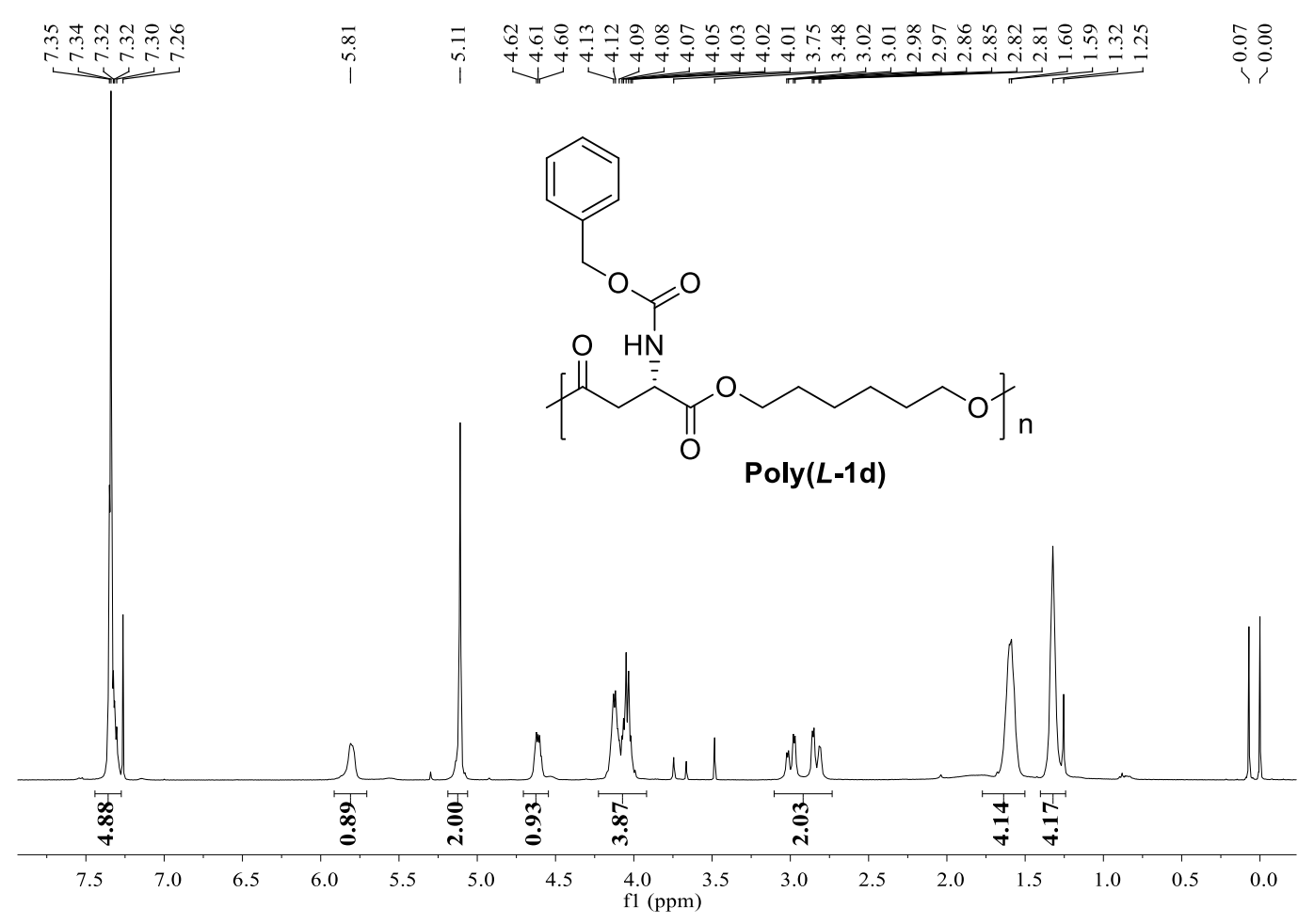

Figure S6. ${ }^{1} \mathrm{H}-\mathrm{NMR}$ spectrum of Poly $(\boldsymbol{L}-\mathbf{1 d})$

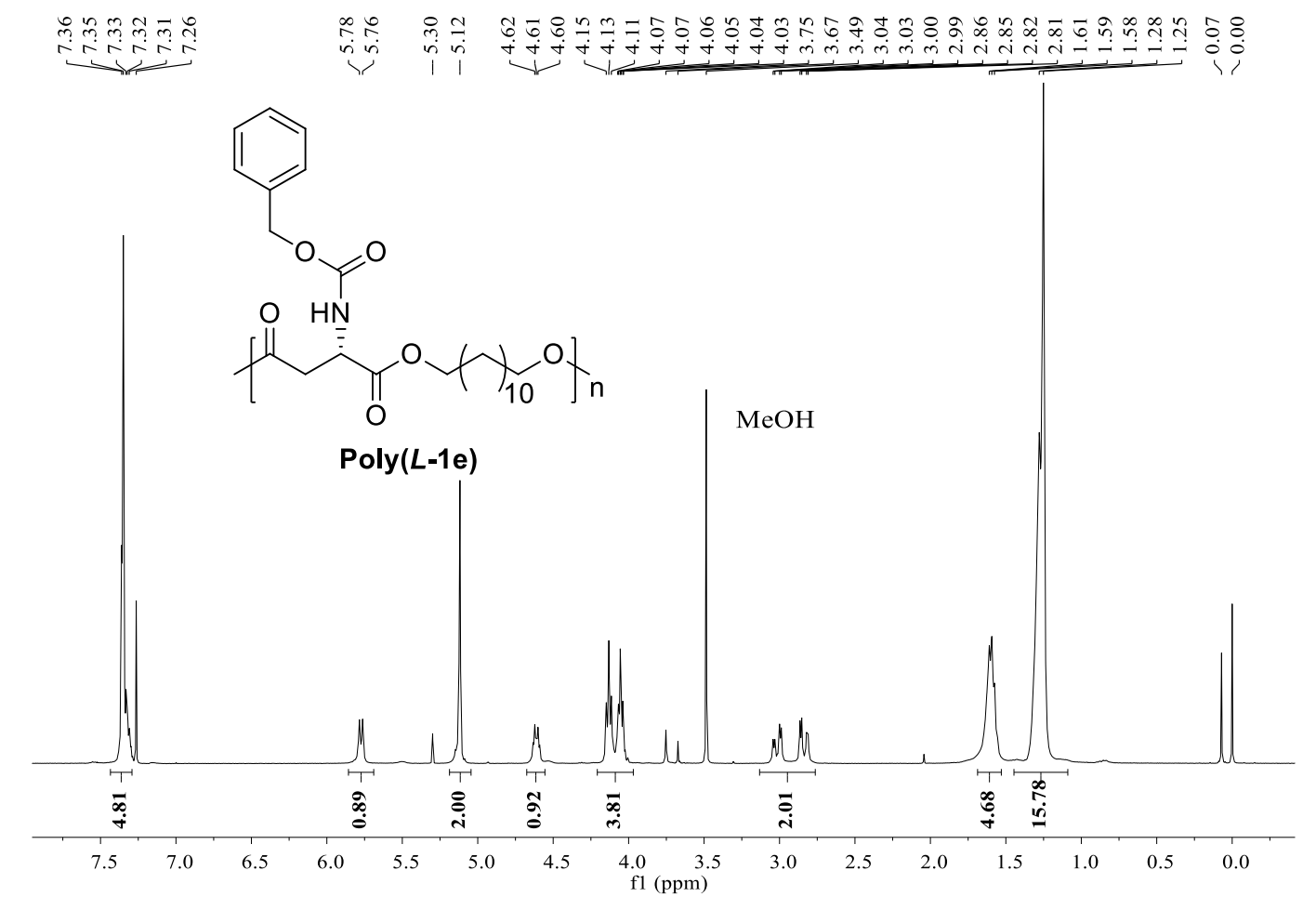

Figure S7. ${ }^{1} \mathrm{H}-\mathrm{NMR}$ spectrum of Poly $(\boldsymbol{L}-1 \mathbf{e})$ 


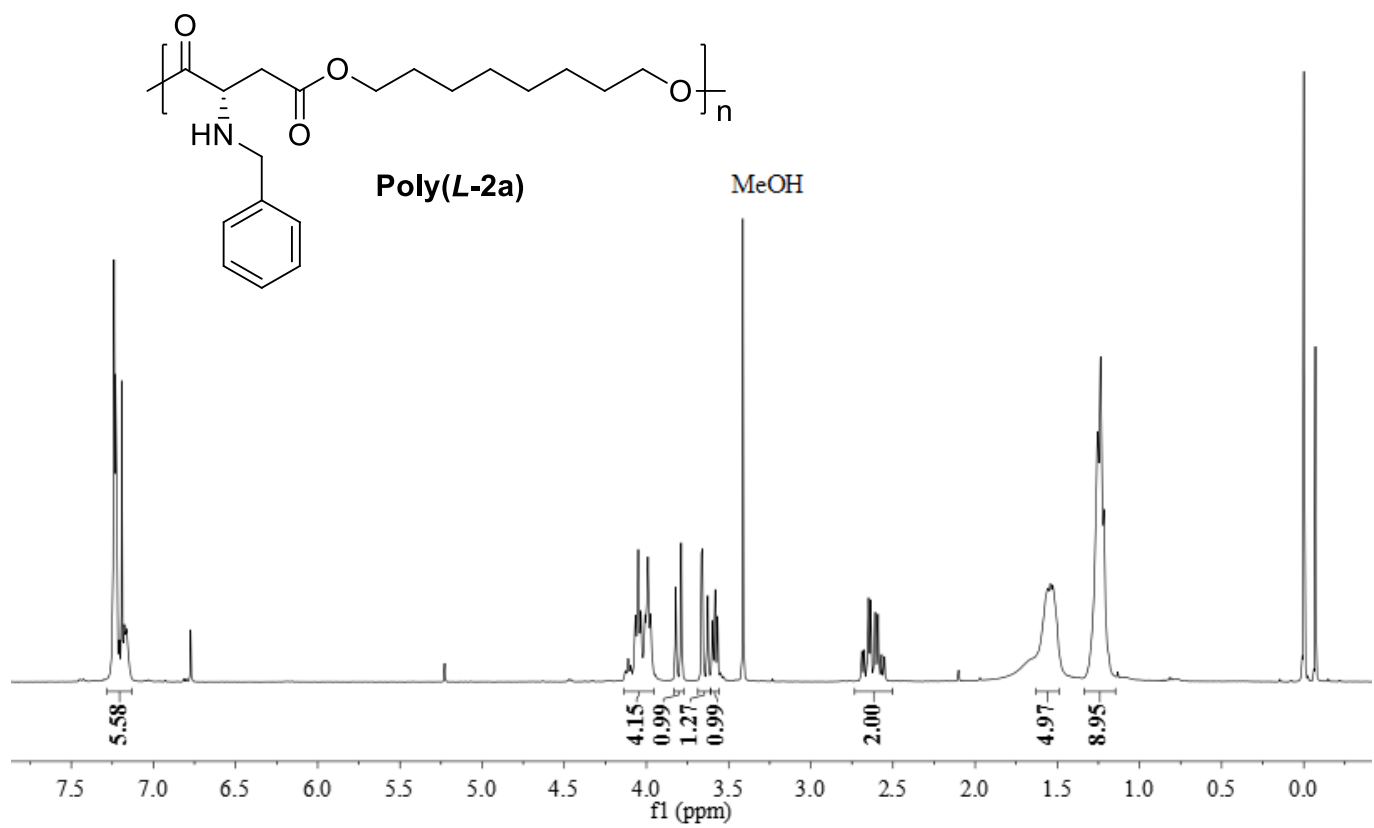

Figure S8. ${ }^{1} \mathrm{H}-\mathrm{NMR}$ spectrum of Poly $(\boldsymbol{L}$-2a $)$

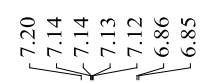

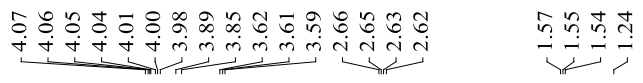
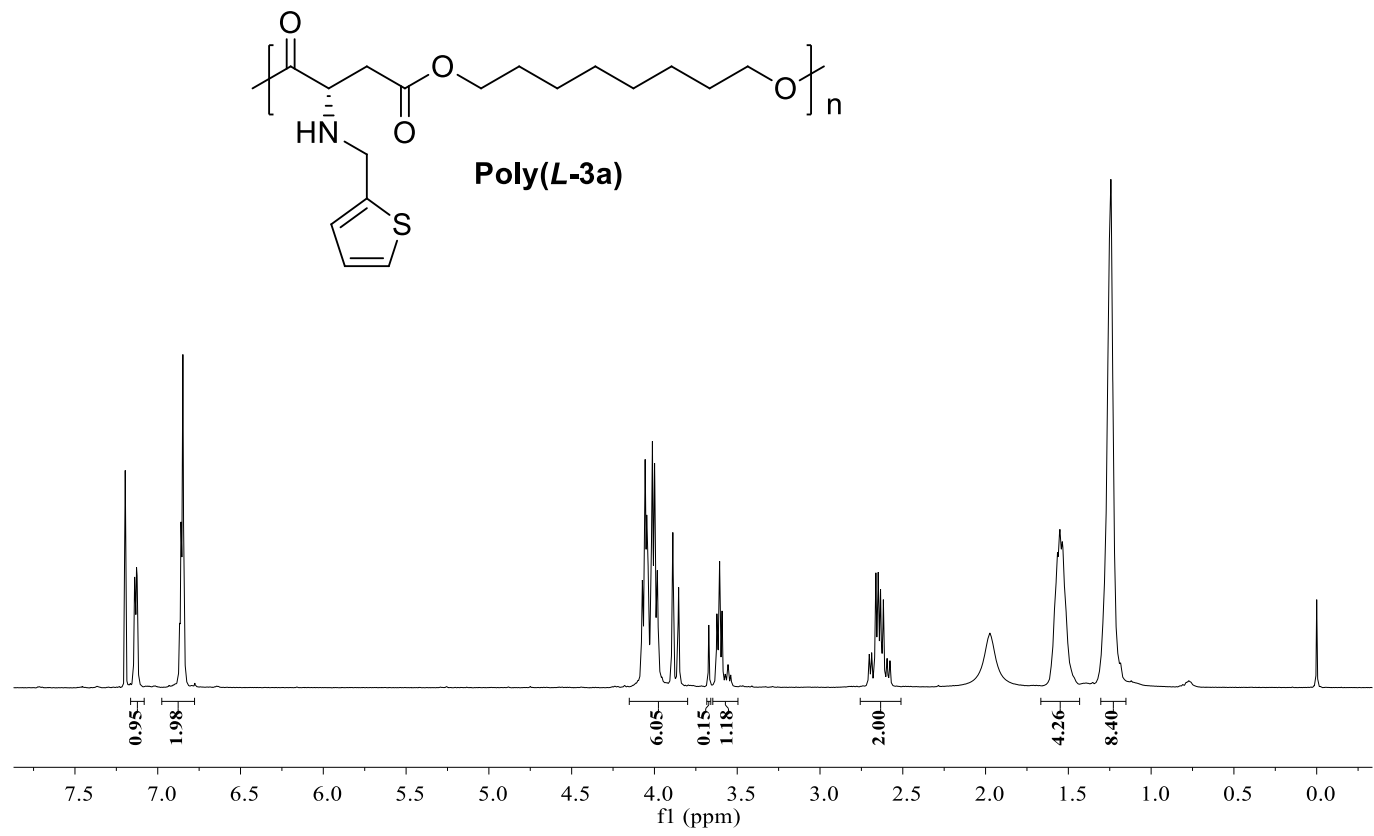

Figure S9. ${ }^{1} \mathrm{H}$-NMR spectrum of Poly $(\boldsymbol{L}-\mathbf{3 a})$ 


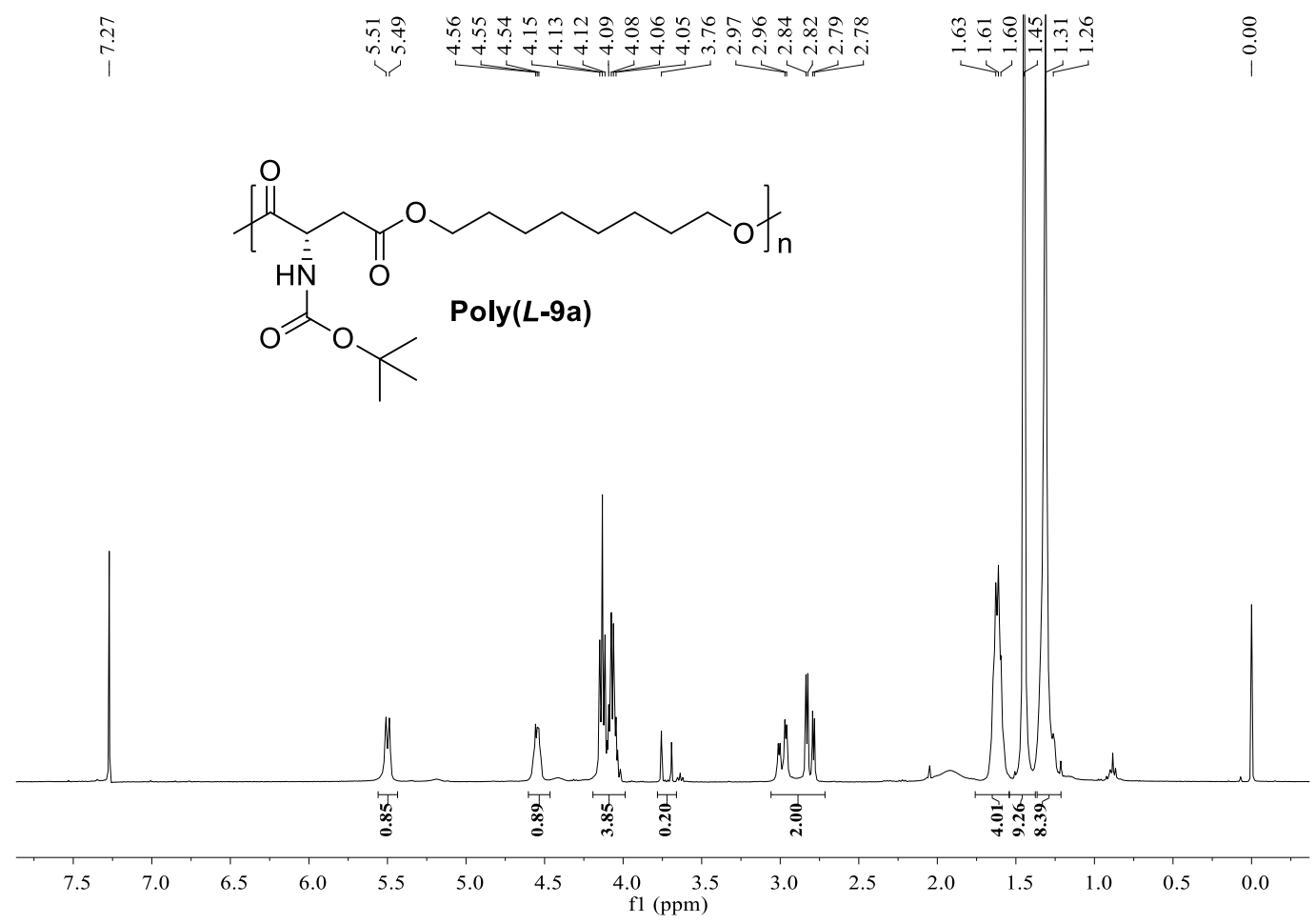

Figure S10. ${ }^{1} \mathrm{H}-\mathrm{NMR}$ spectrum of Poly $(\boldsymbol{L}$-9a)

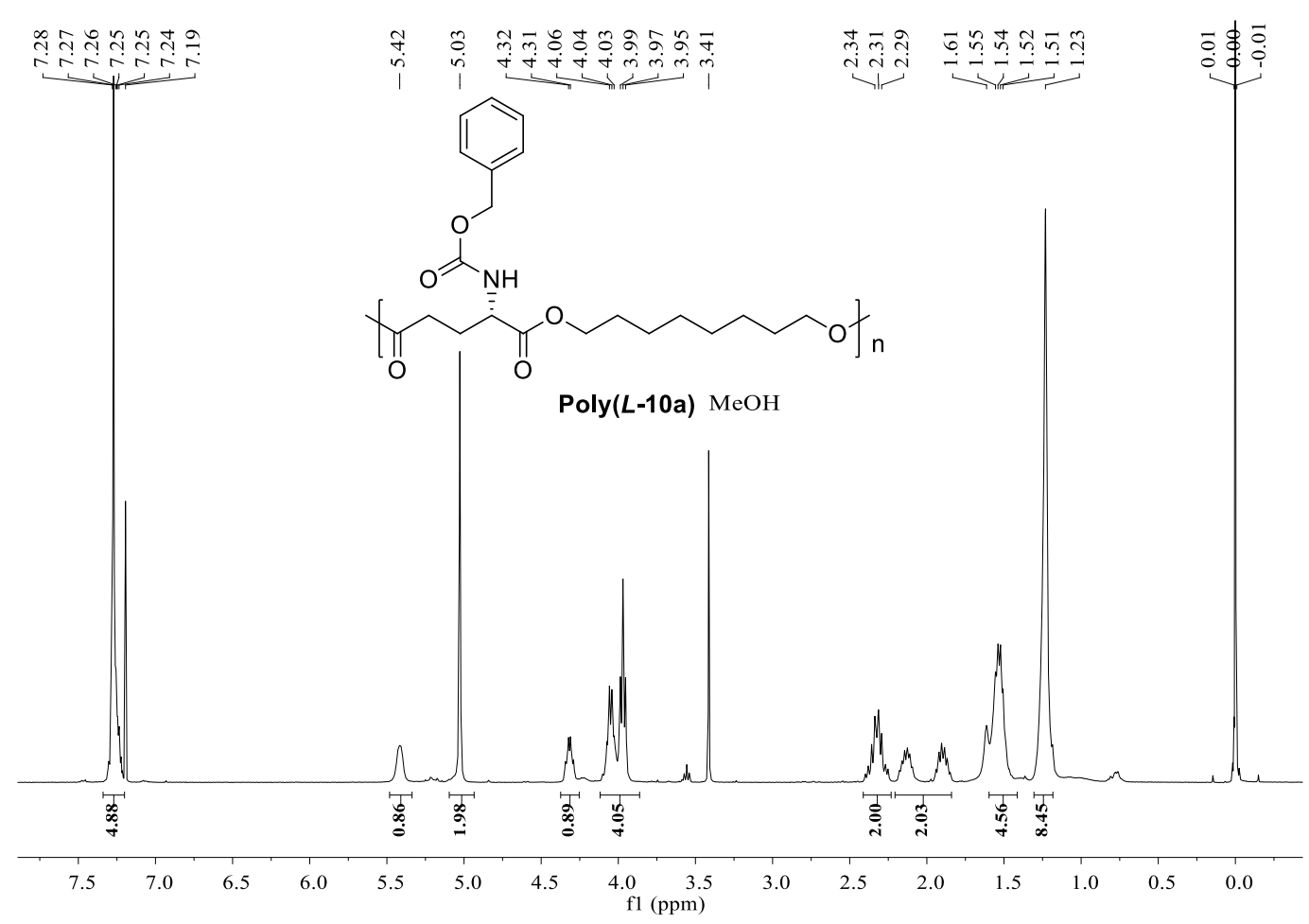

Figure S11. ${ }^{1}$ H-NMR spectrum of Poly $(L-10 a)$ 


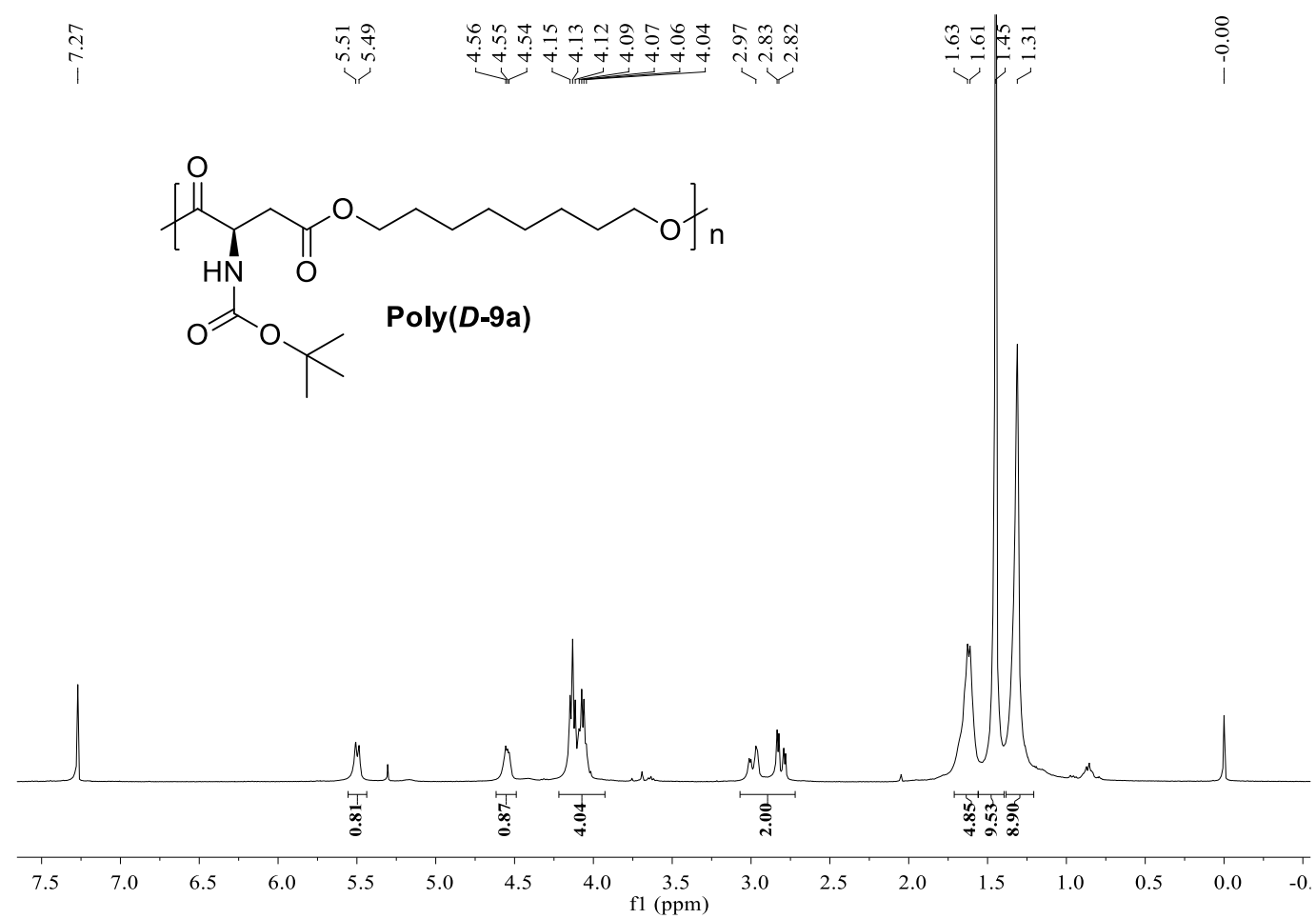

Figure S12. ${ }^{1} \mathrm{H}-\mathrm{NMR}$ spectrum of Poly (D-9a) 
3. ${ }^{1} \mathrm{H}$ and ${ }^{13} \mathrm{C}$ NMR spectra of monomers
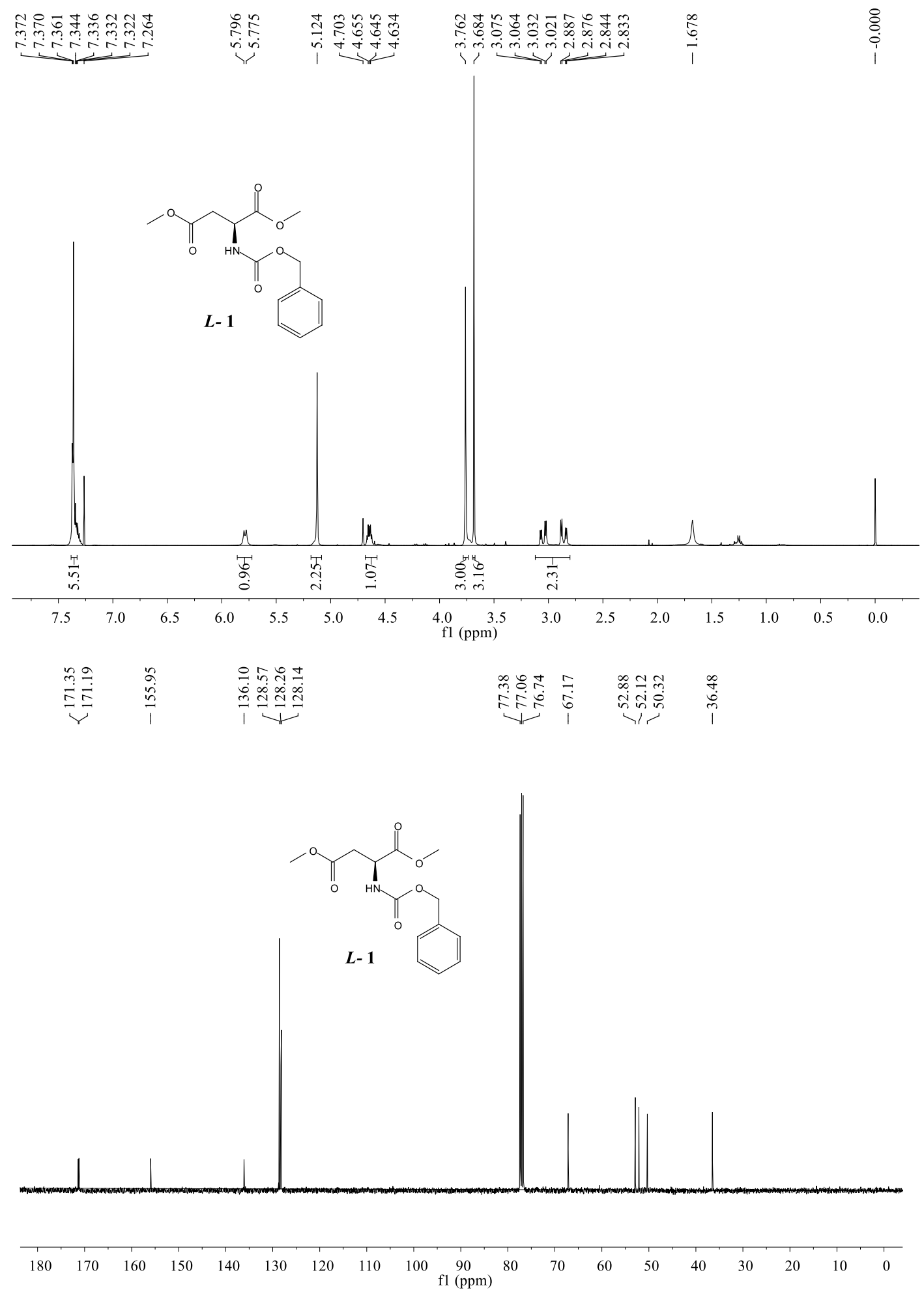

Figure S13. ${ }^{1} \mathrm{H}$ and ${ }^{13} \mathrm{C}$ NMR spectra of $\boldsymbol{L}-\mathbf{1}$ 

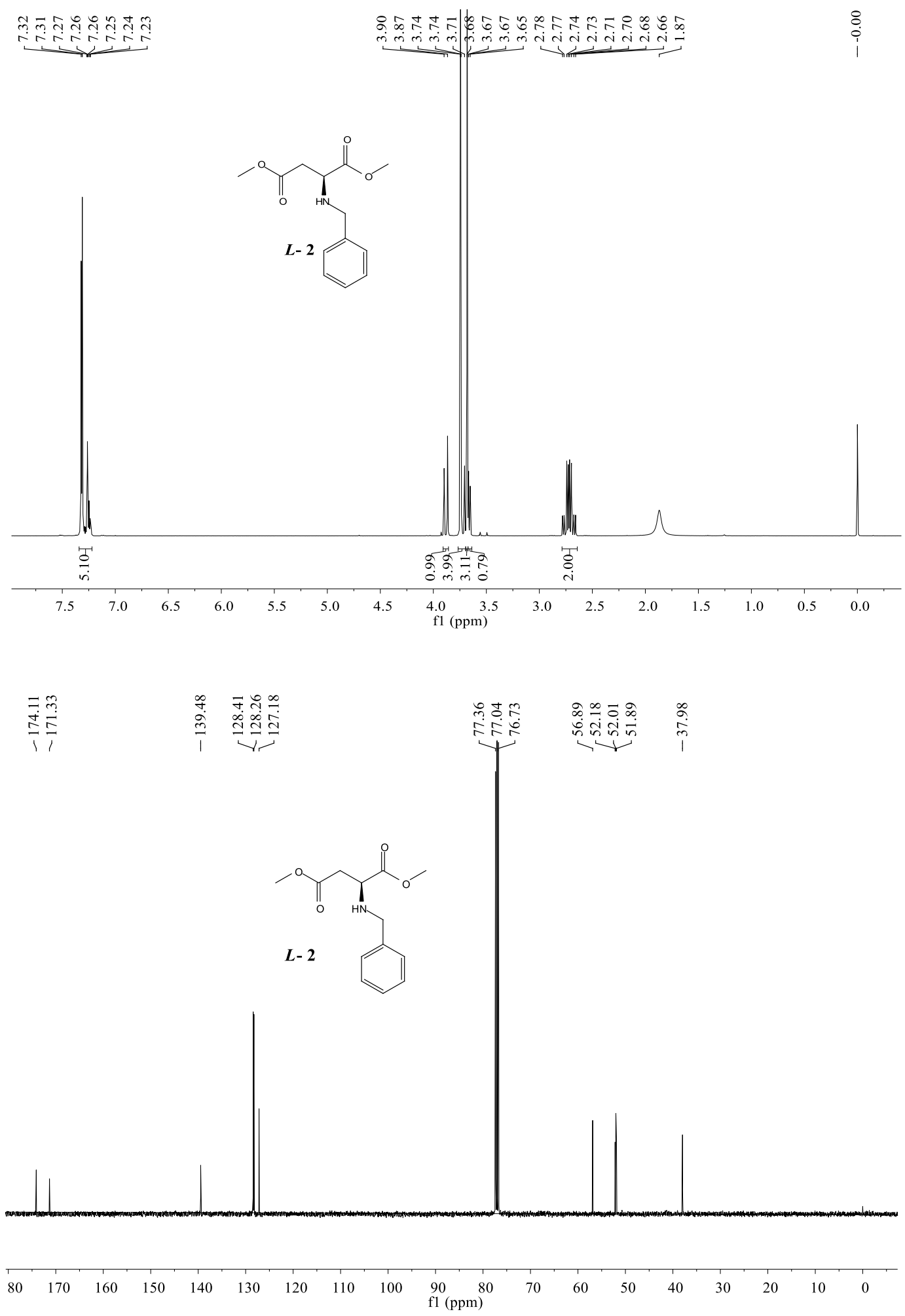

Figure S14. ${ }^{1} \mathrm{H}$ and ${ }^{13} \mathrm{C}$ NMR spectra of $\boldsymbol{L}-\mathbf{2}$ 


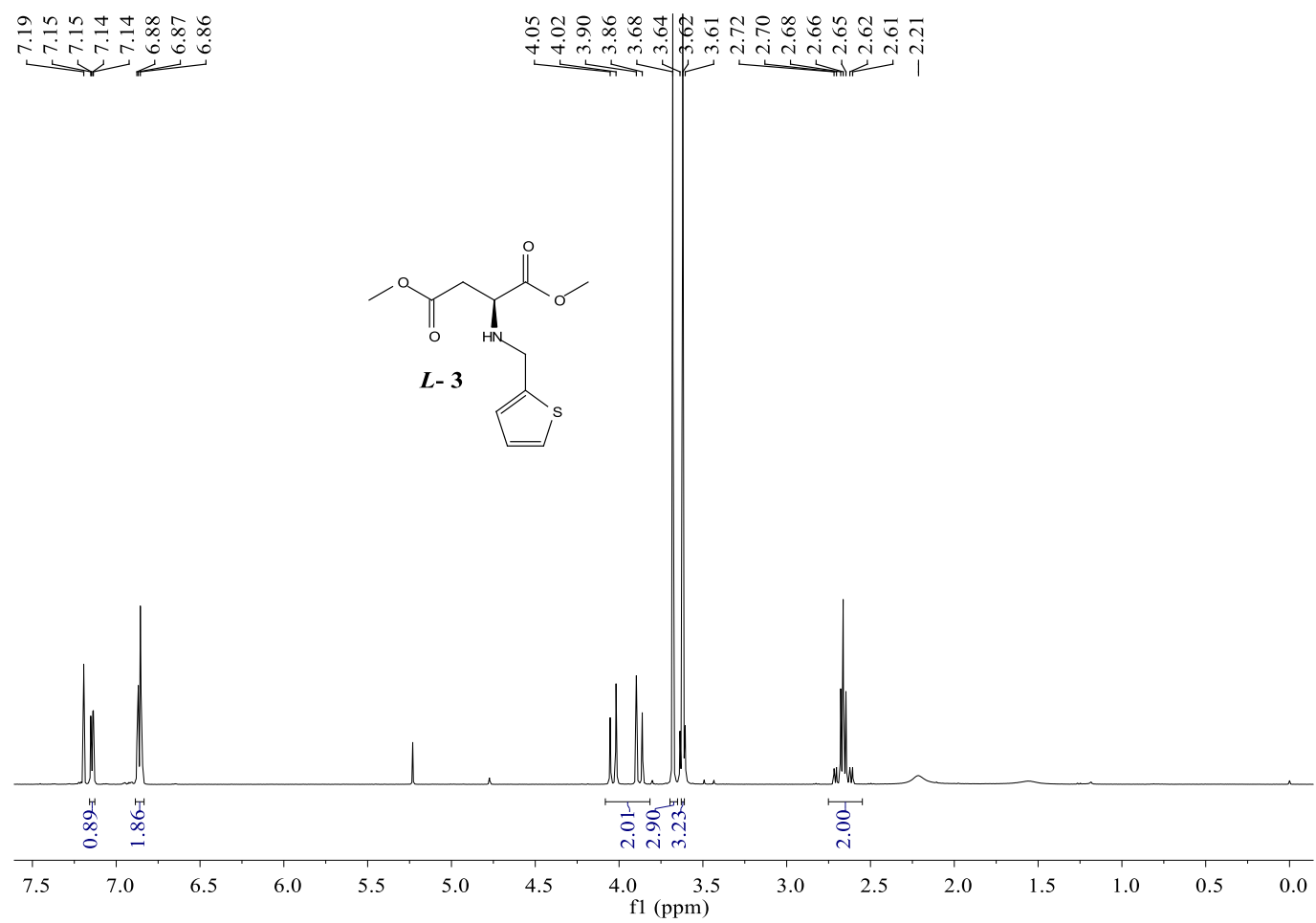

\begin{tabular}{|c|c|c|c|c|}
\hline 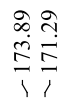 & $\begin{array}{l}\stackrel{2}{\tilde{y}} \\
\text { I }\end{array}$ & 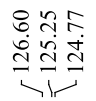 & 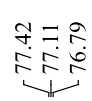 & 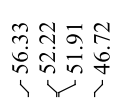 \\
\hline
\end{tabular}

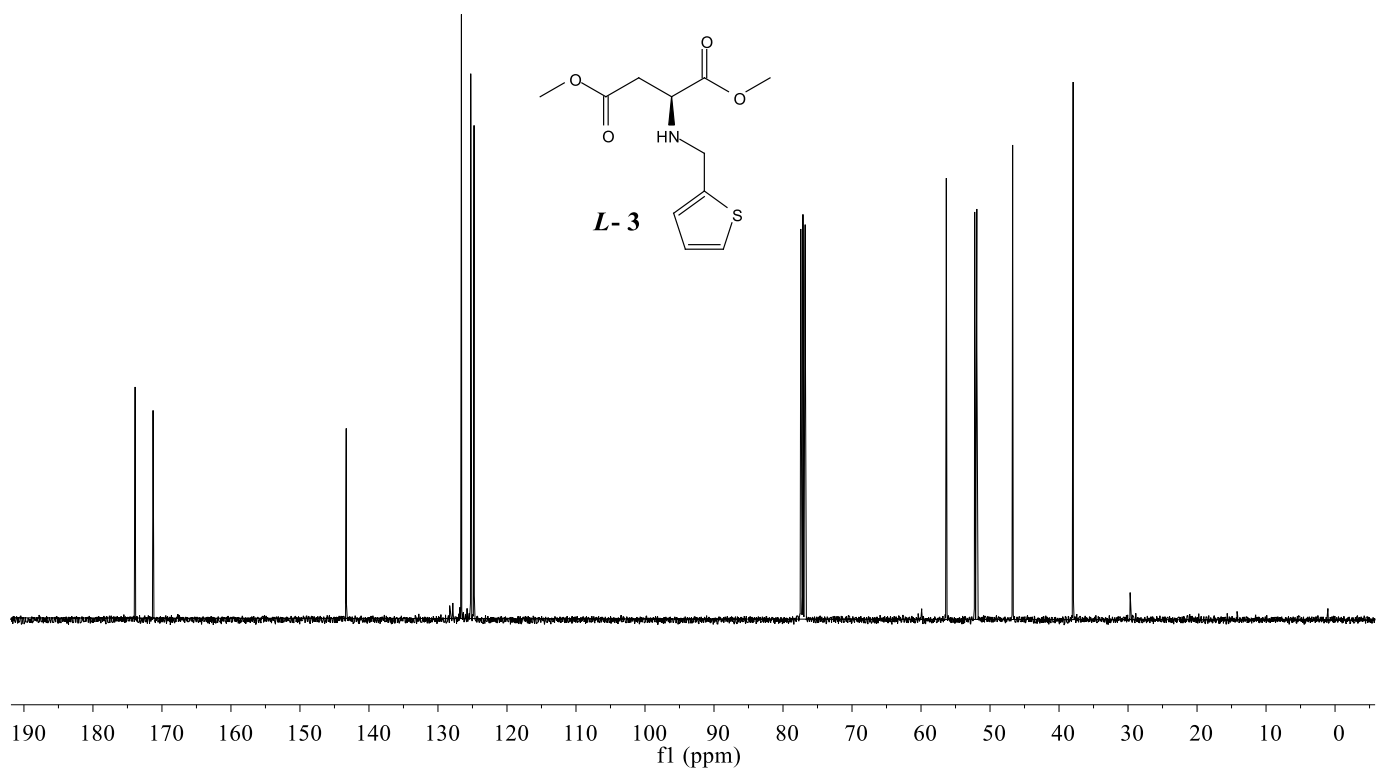

Figure S15. ${ }^{1} \mathrm{H}$ and ${ }^{13} \mathrm{C}$ NMR spectra of $\boldsymbol{L - 3}$ 


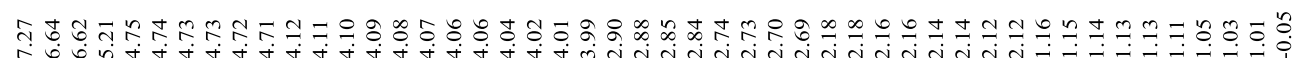
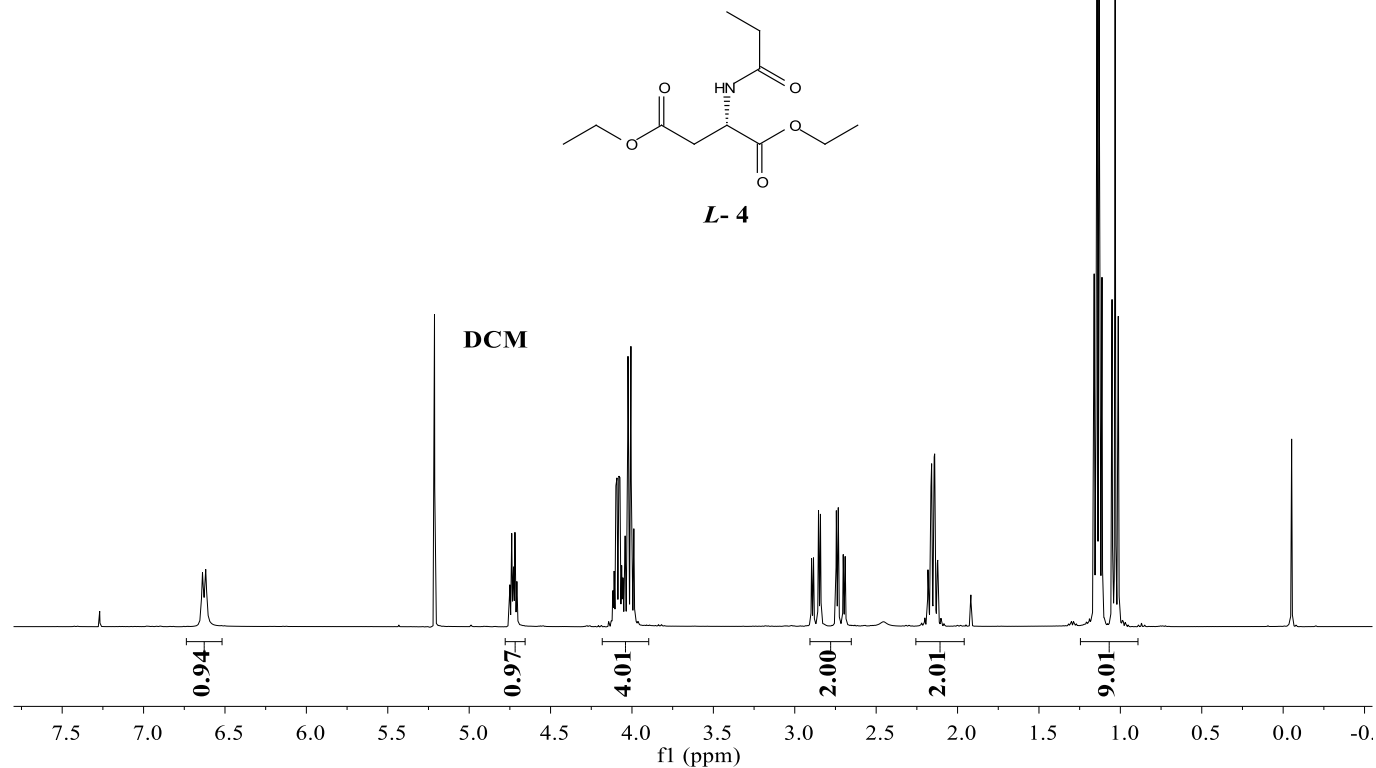

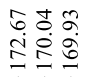

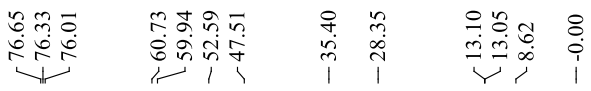

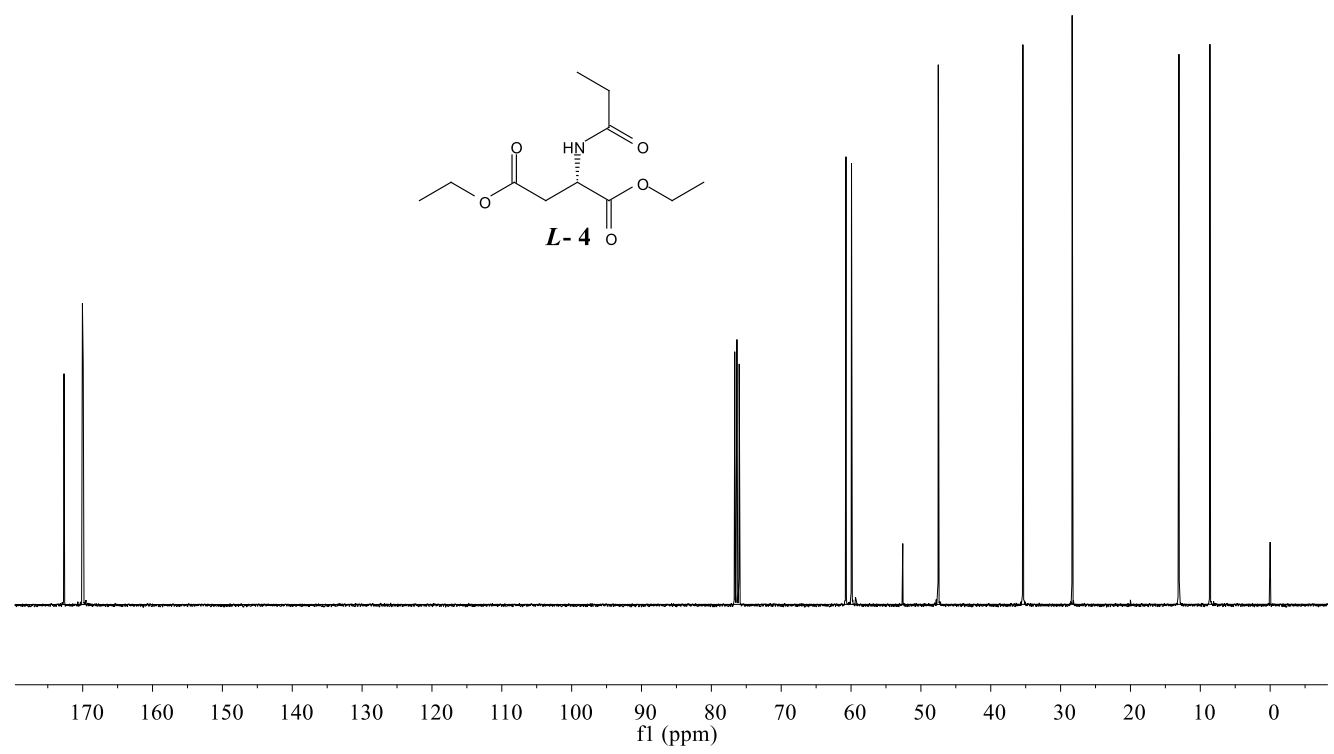

Figure S16. ${ }^{1} \mathrm{H}$ and ${ }^{13} \mathrm{C}$ NMR spectra of $\boldsymbol{L}-\mathbf{4}$ 


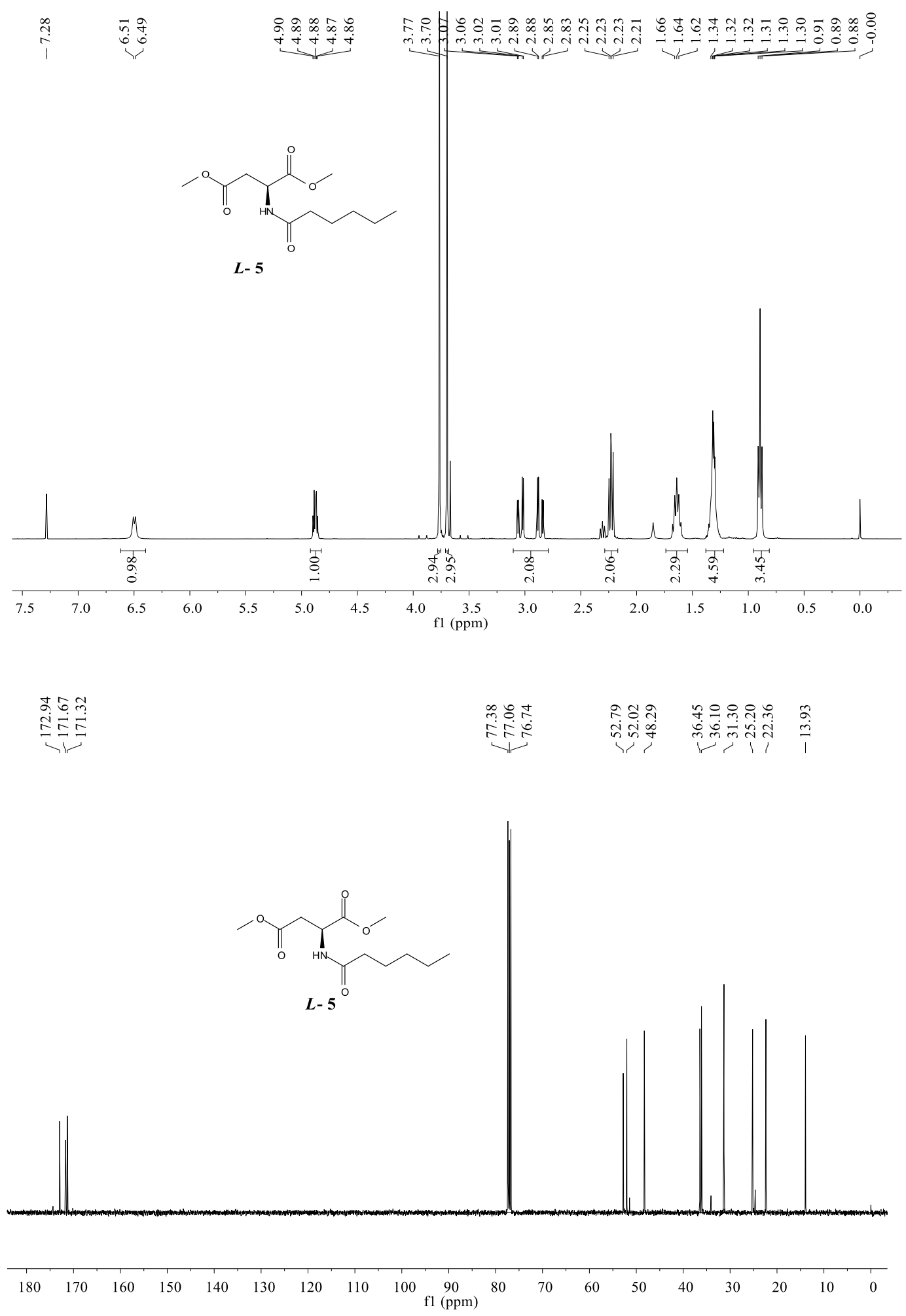

Figure S17. ${ }^{1} \mathrm{H}$ and ${ }^{13} \mathrm{C}$ NMR spectra of $\boldsymbol{L}-5$ 


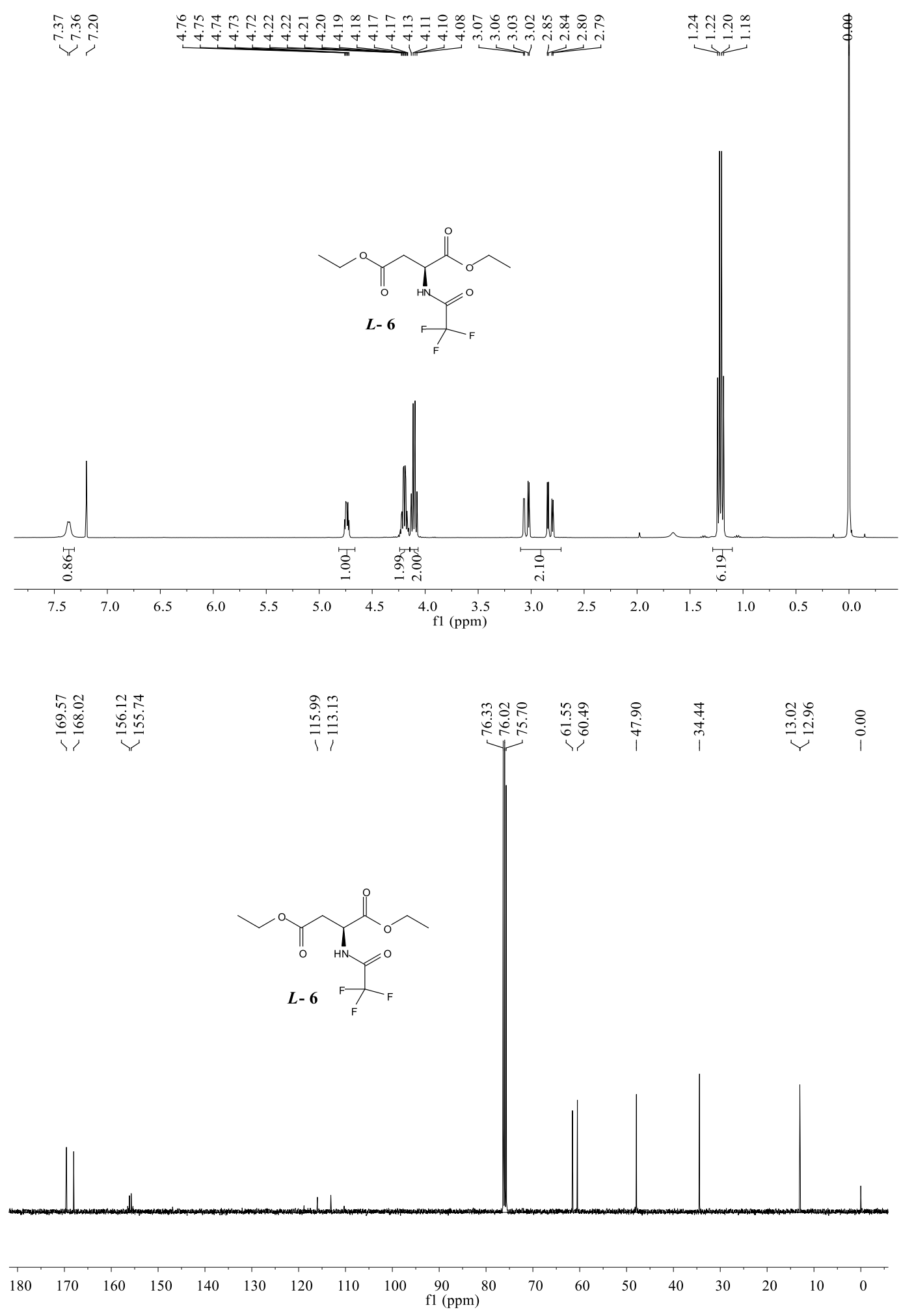

Figure S18. ${ }^{1} \mathrm{H}$ and ${ }^{13} \mathrm{C}$ NMR spectra of $\boldsymbol{L}-6$ 

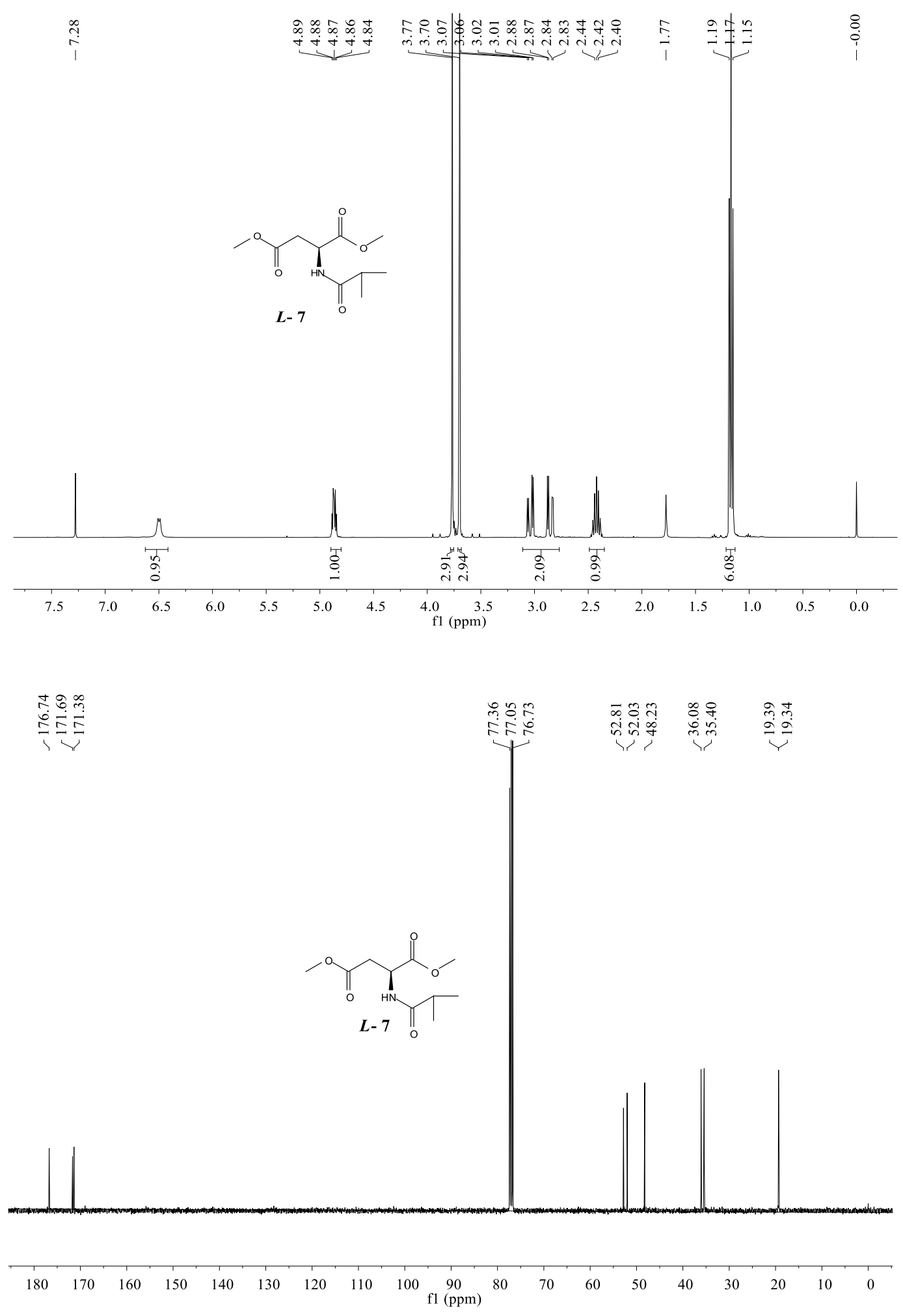

Figure S19. ${ }^{1} \mathrm{H}$ and ${ }^{13} \mathrm{C}$ NMR spectra of $\boldsymbol{L}-\mathbf{7}$ 

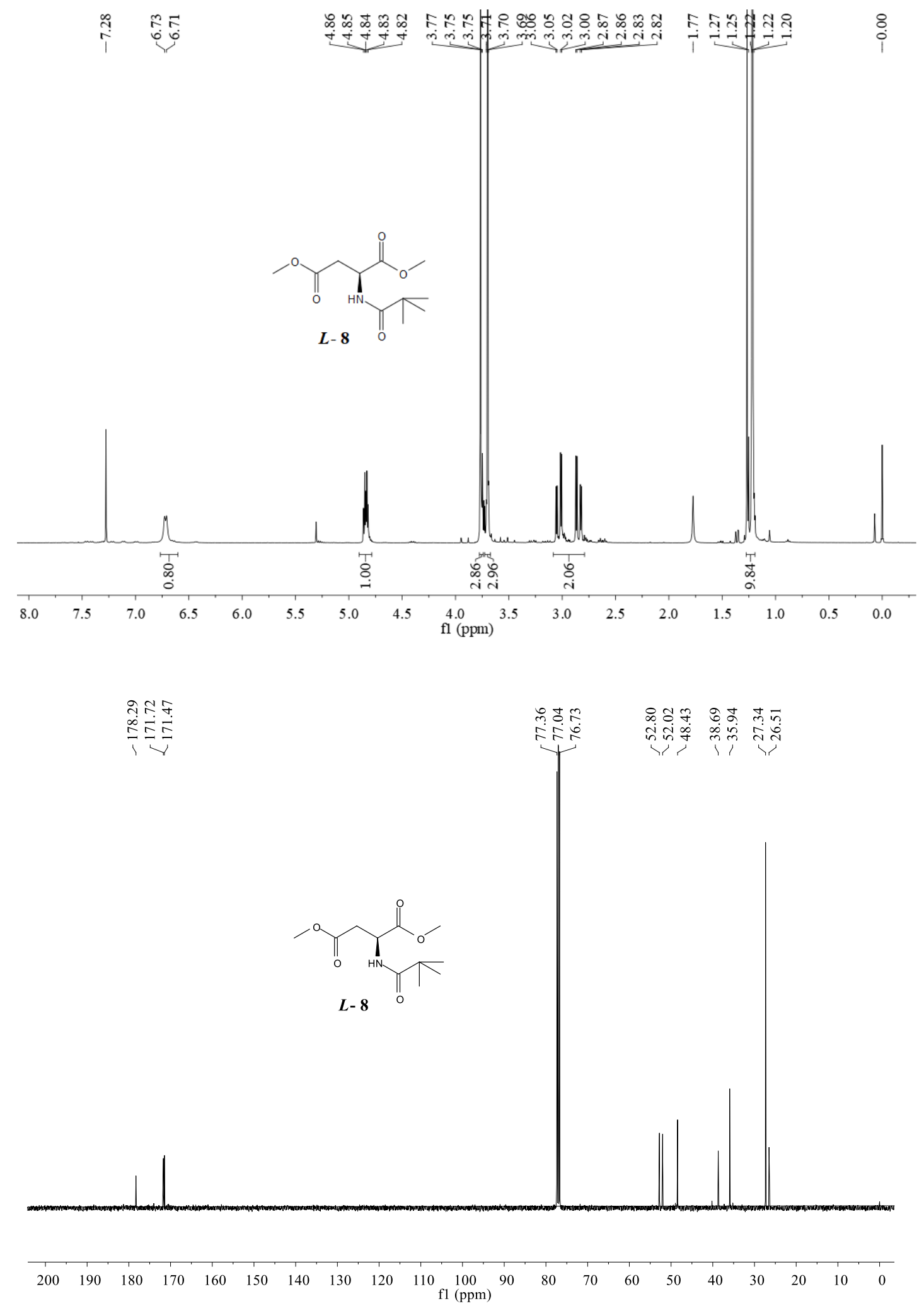

Figure S20. ${ }^{1} \mathrm{H}$ and ${ }^{13} \mathrm{C}$ NMR spectra of $\boldsymbol{L}-\mathbf{8}$ 

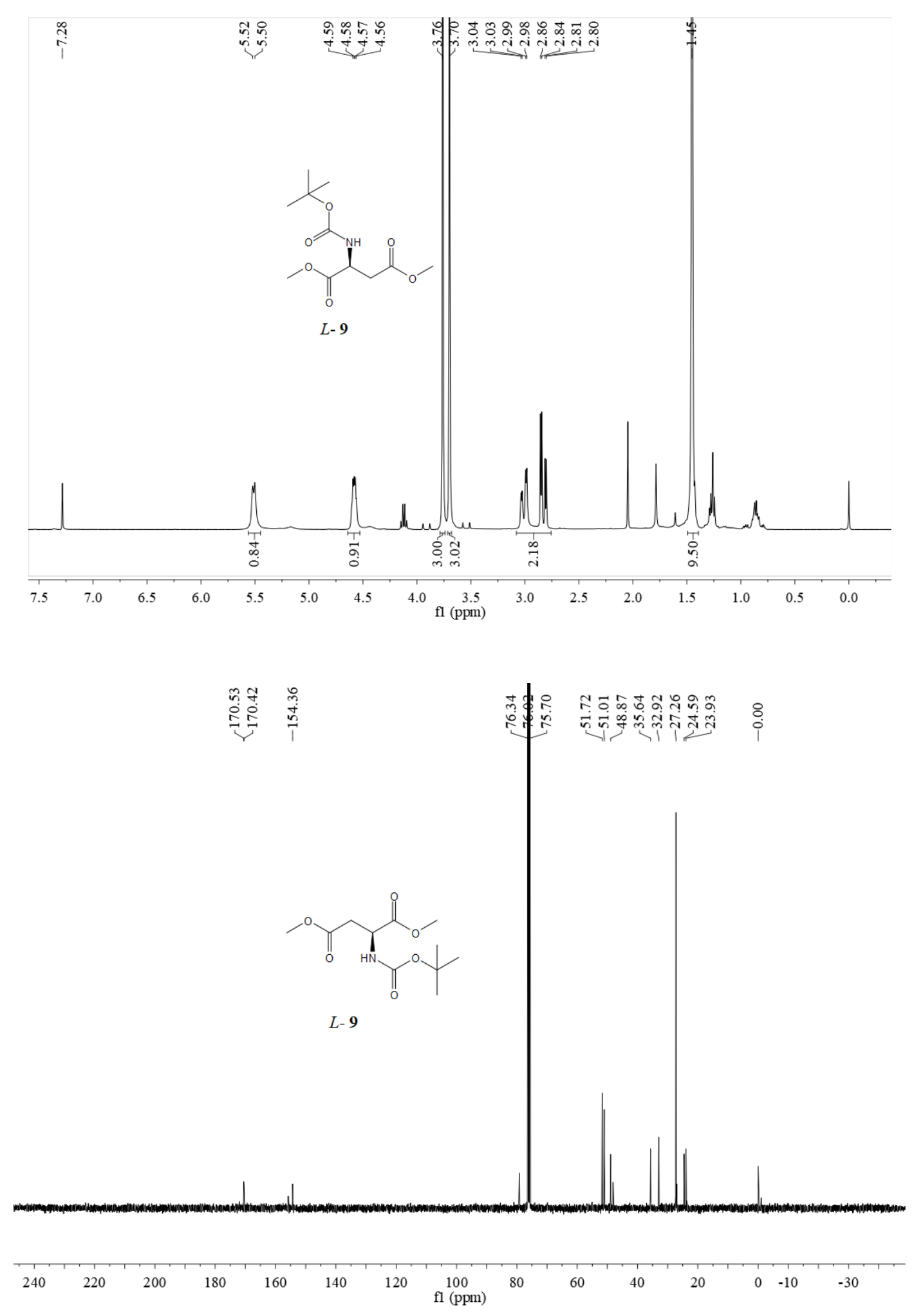

Figure S21. ${ }^{1} \mathrm{H}$ and ${ }^{13} \mathrm{C}$ NMR spectra of $\boldsymbol{L}-\mathbf{9}$ 

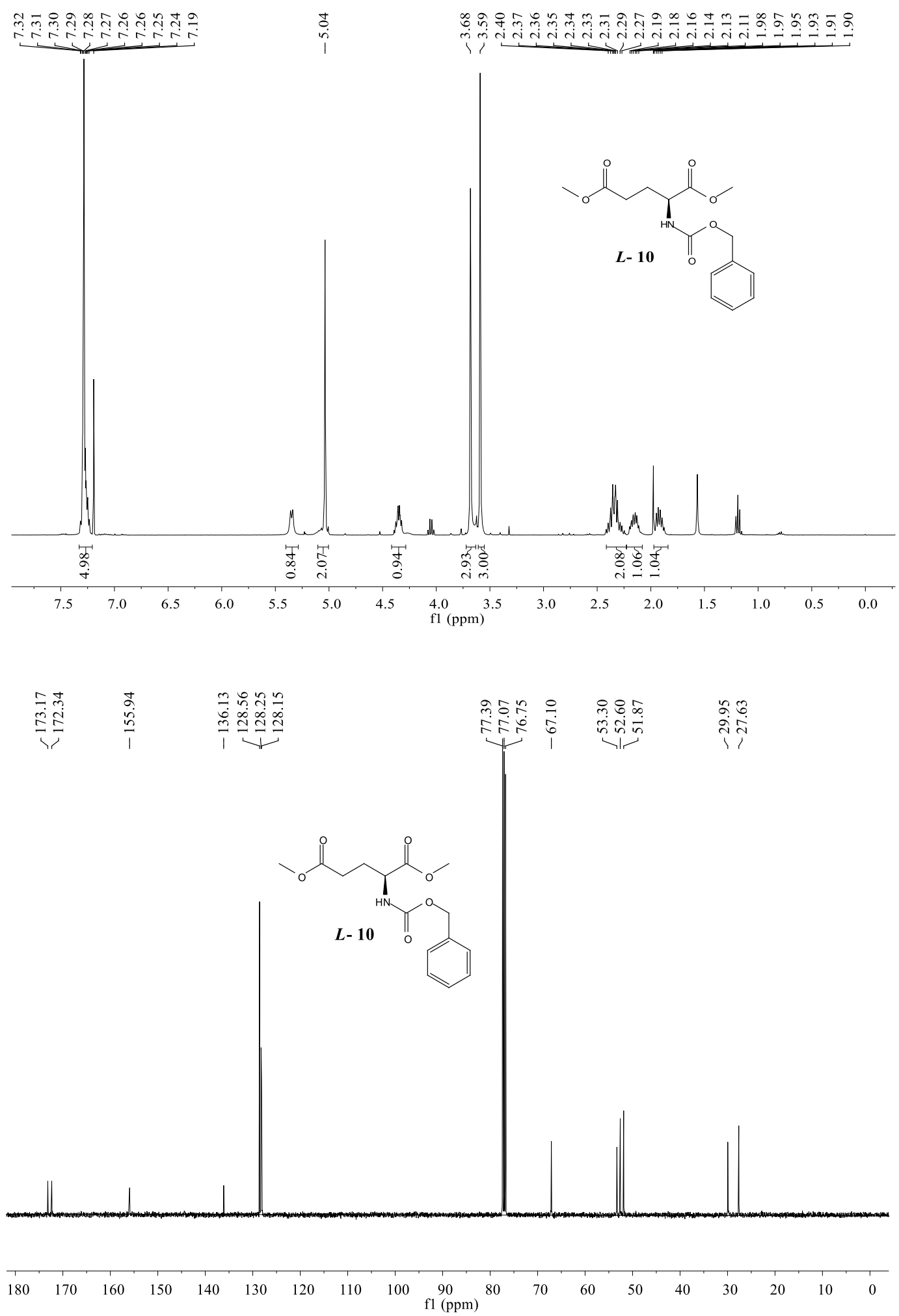

Figure S22. ${ }^{1} \mathrm{H}$ and ${ }^{13} \mathrm{C}$ NMR spectra of $\boldsymbol{L}-\mathbf{1 0}$ 


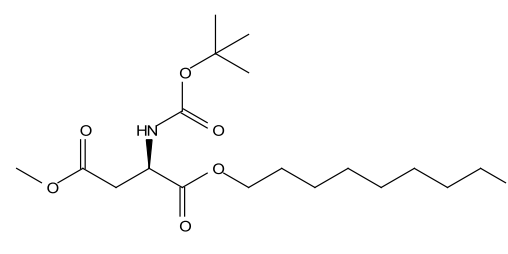

D- 11
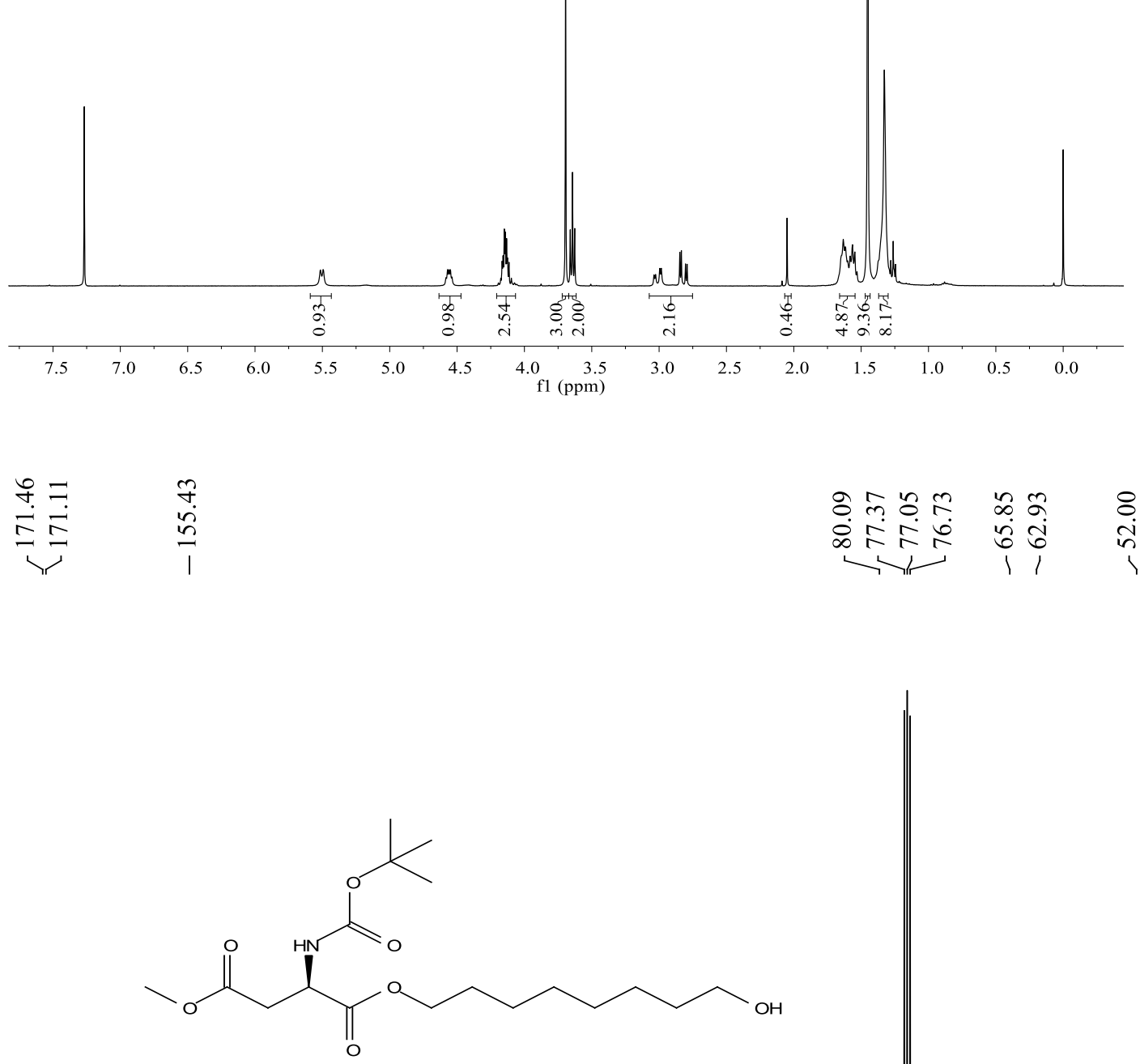

D- 11

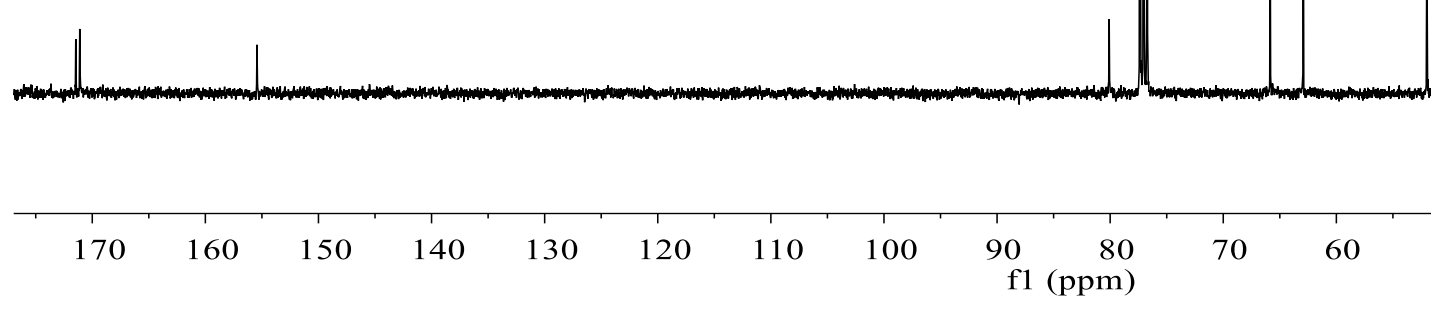

Figure S23. ${ }^{1} \mathrm{H}$ and ${ }^{13} \mathrm{C}$ NMR spectra of $\boldsymbol{D}-\mathbf{1 1}$ 

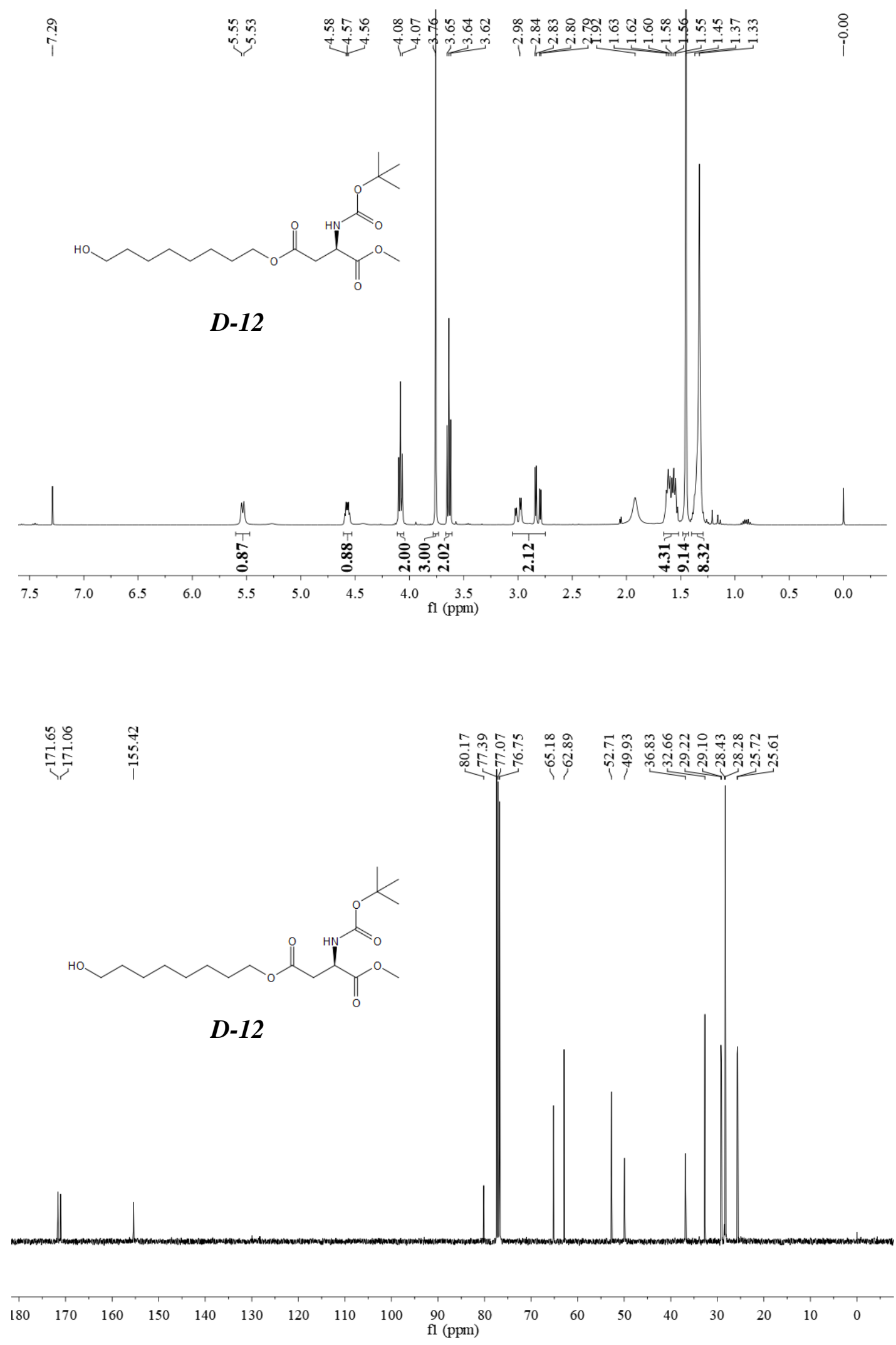

Figure S24. ${ }^{1} \mathrm{H}$ and ${ }^{13} \mathrm{C}$ NMR spectra of $\boldsymbol{D}-\mathbf{1 2}$ 


\section{SEC of chiral polyesters}

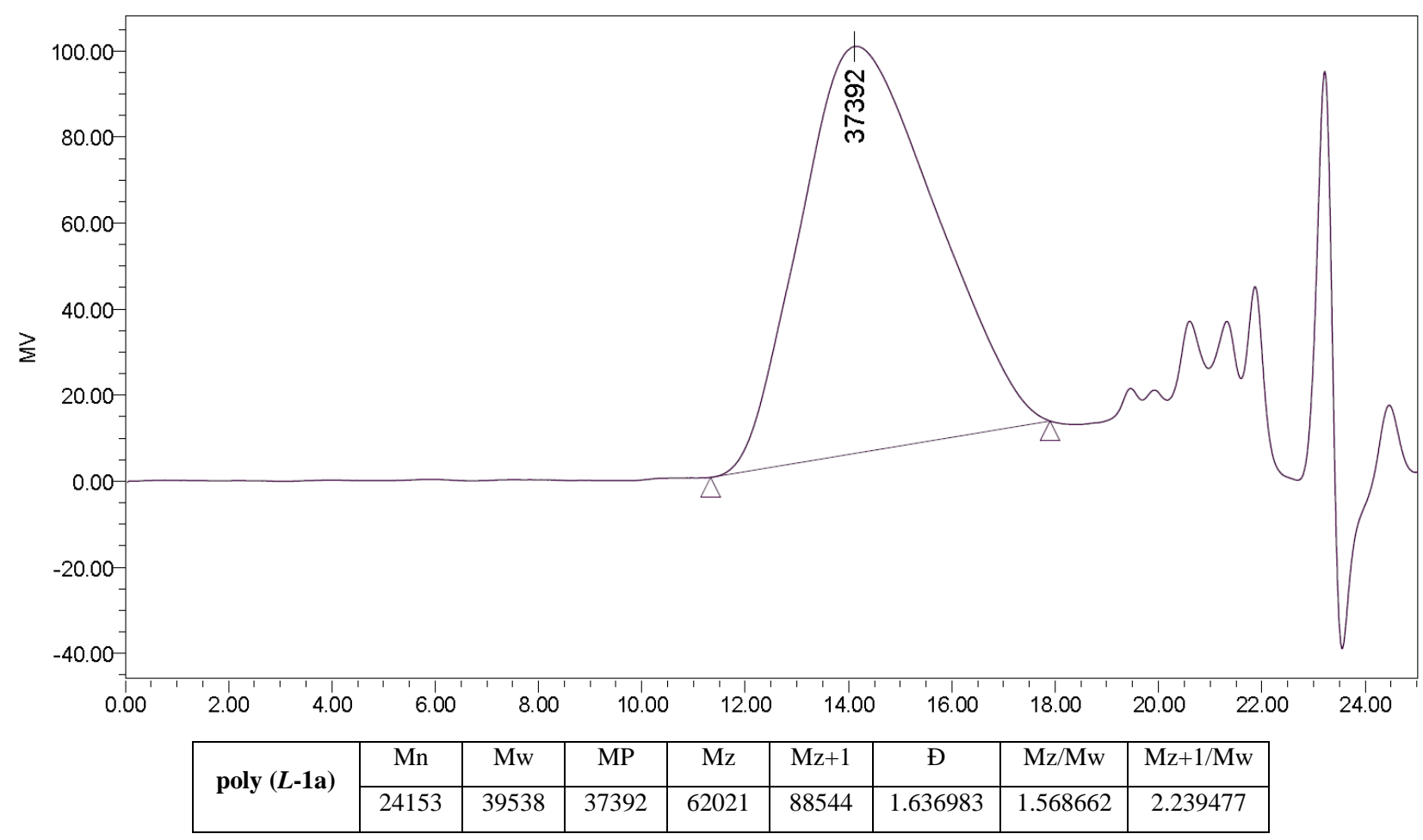

Figure S25. SEC data of Poly $(\boldsymbol{L}-\mathbf{- 1 a})$

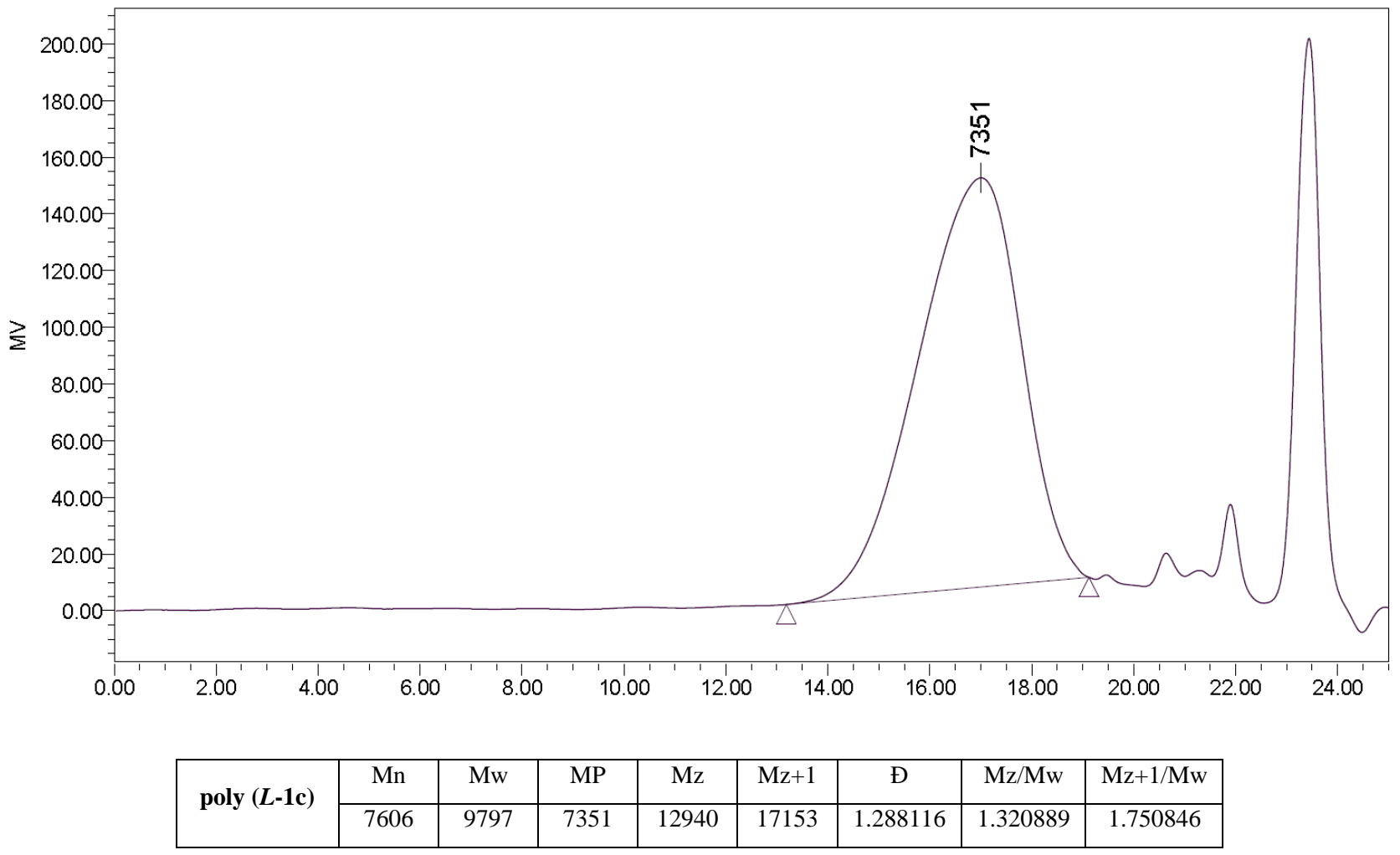

Figure S26. SEC data of Poly $(L-1 c)$ 


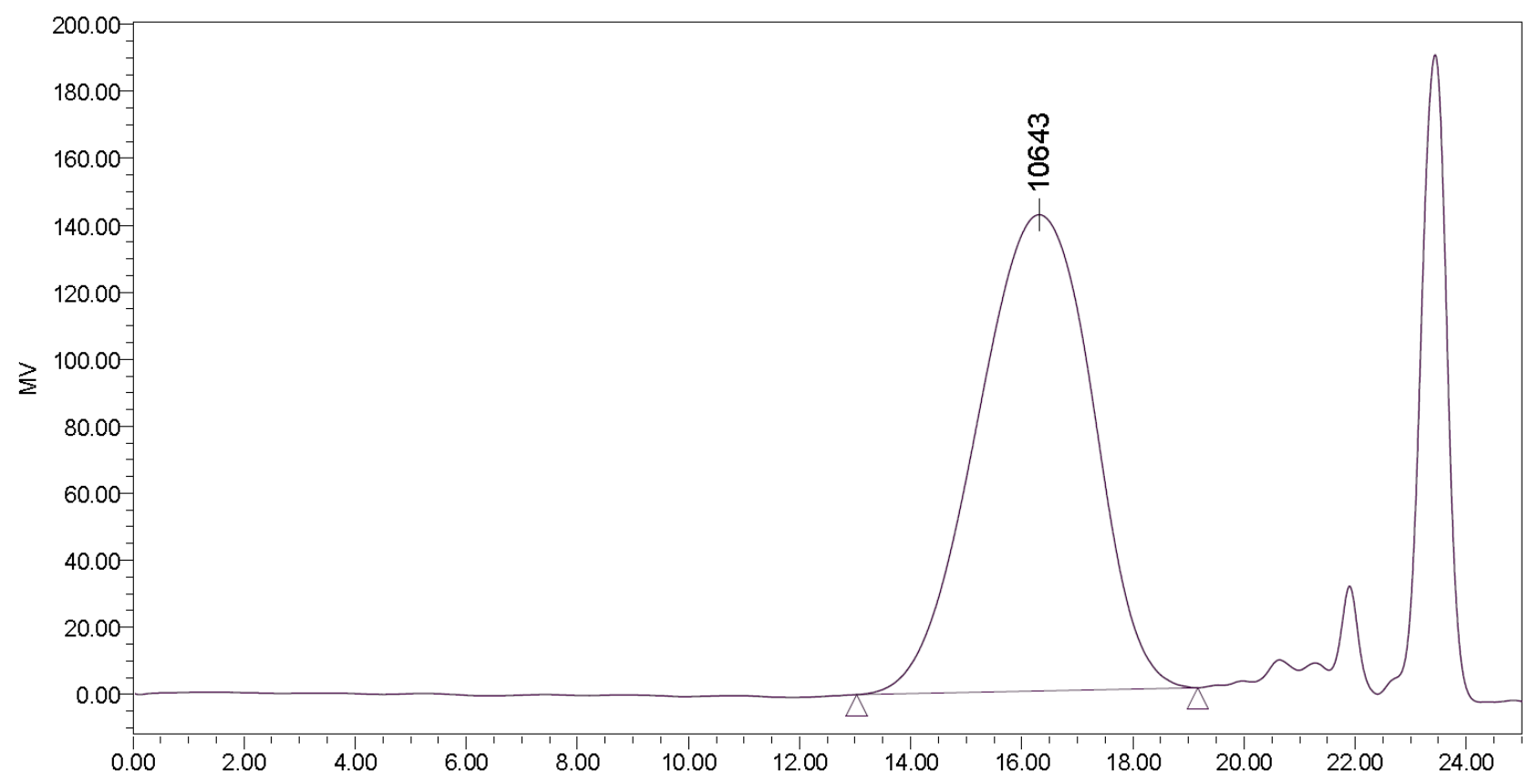

\begin{tabular}{|c|c|c|c|c|c|c|c|c|}
\hline \multirow{2}{*}{ poly $(\boldsymbol{L}-1 \mathbf{1 d})$} & $\mathrm{Mn}$ & $\mathrm{Mw}$ & $\mathrm{MP}$ & $\mathrm{Mz}$ & $\mathrm{Mz}+1$ & $Ð$ & $\mathrm{Mz} / \mathrm{Mw}$ & $\mathrm{Mz}+1 / \mathrm{Mw}$ \\
\cline { 2 - 9 } & 9961 & 12817 & 10643 & 16695 & 21628 & 1.286702 & 1.302505 & 1.687395 \\
\hline
\end{tabular}

Figure S27. SEC data of Poly $(L-1 d)$

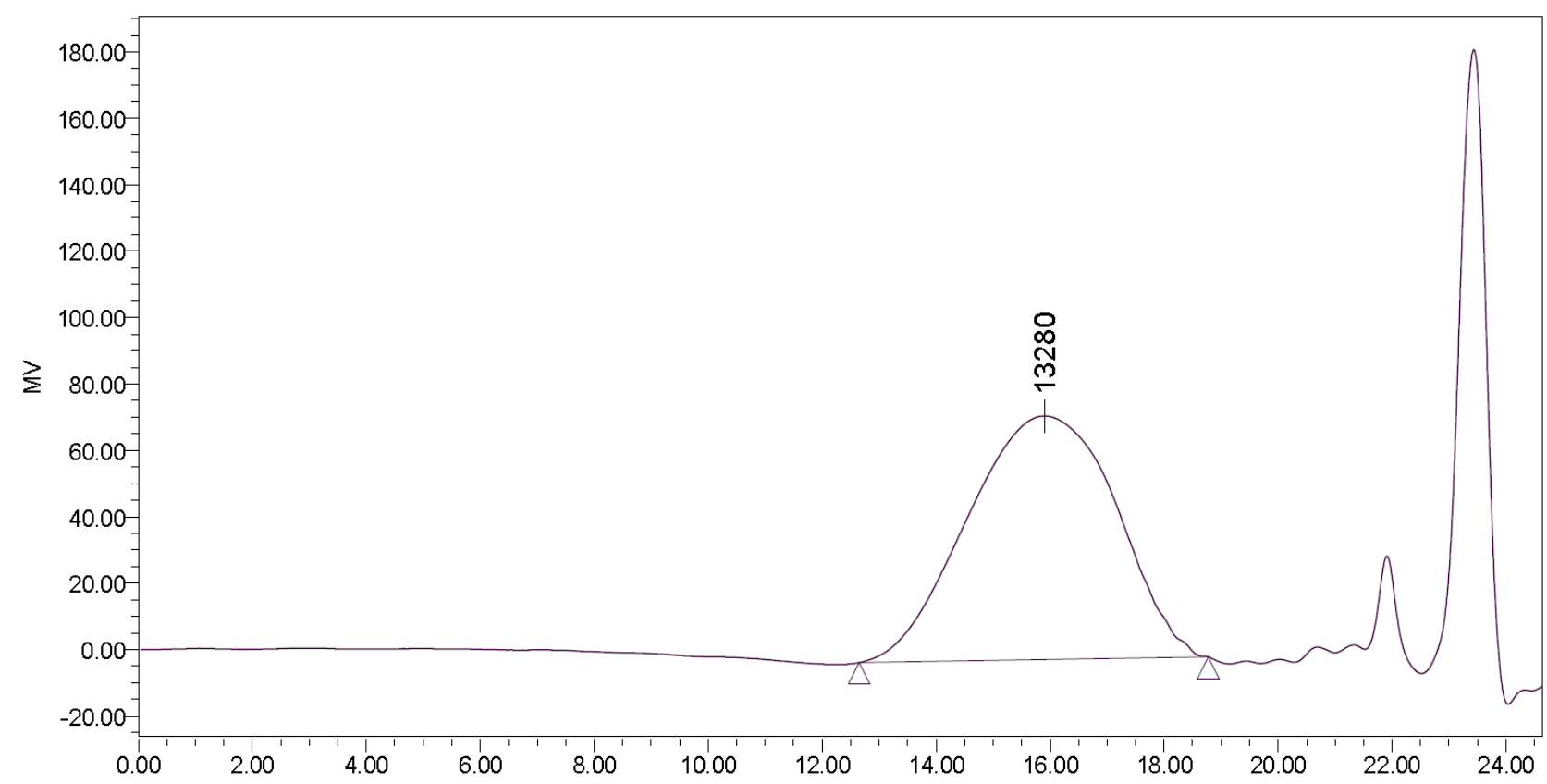

poly $(L-1 \mathrm{e})$

\begin{tabular}{|c|c|c|c|c|c|c|c|}
\hline $\mathrm{Mn}$ & $\mathrm{Mw}$ & $\mathrm{MP}$ & $\mathrm{Mz}$ & $\mathrm{Mz}+1$ & $\mathrm{D}$ & $\mathrm{Mz} / \mathrm{Mw}$ & $\mathrm{Mz}+1 / \mathrm{Mw}$ \\
\hline 11653 & 16920 & 13280 & 24784 & 34549 & 1.451934 & 1.464768 & 2.041959 \\
\hline
\end{tabular}

Figure S28. SEC data of Poly $(\boldsymbol{L}-1 \mathrm{e})$ 


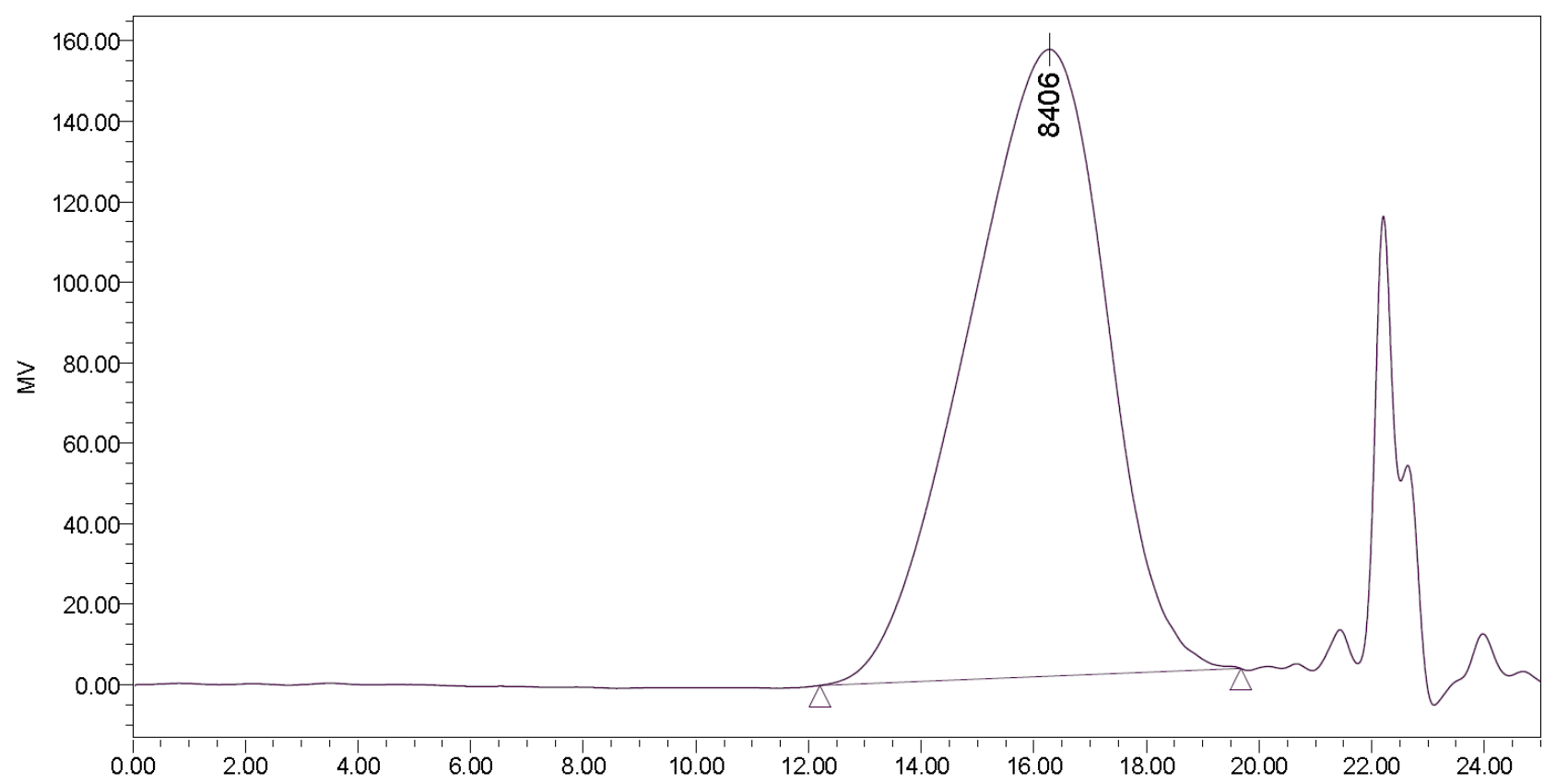

\begin{tabular}{|c|c|c|c|c|c|c|c|c|}
\hline \multirow{2}{*}{ poly $(\boldsymbol{L}$-2a $)$} & $\mathrm{Mn}$ & $\mathrm{Mw}$ & $\mathrm{MP}$ & $\mathrm{Mz}$ & $\mathrm{Mz}+1$ & Đ & Mz/Mw & $\mathrm{Mz}+1 / \mathrm{Mw}$ \\
\cline { 2 - 9 } & 8118 & 12100 & 8406 & 18477 & 27406 & 1.490568 & 1.52953 & 2.264870 \\
\hline
\end{tabular}

Figure S29. SEC data of Poly $(\boldsymbol{L}$-2a)

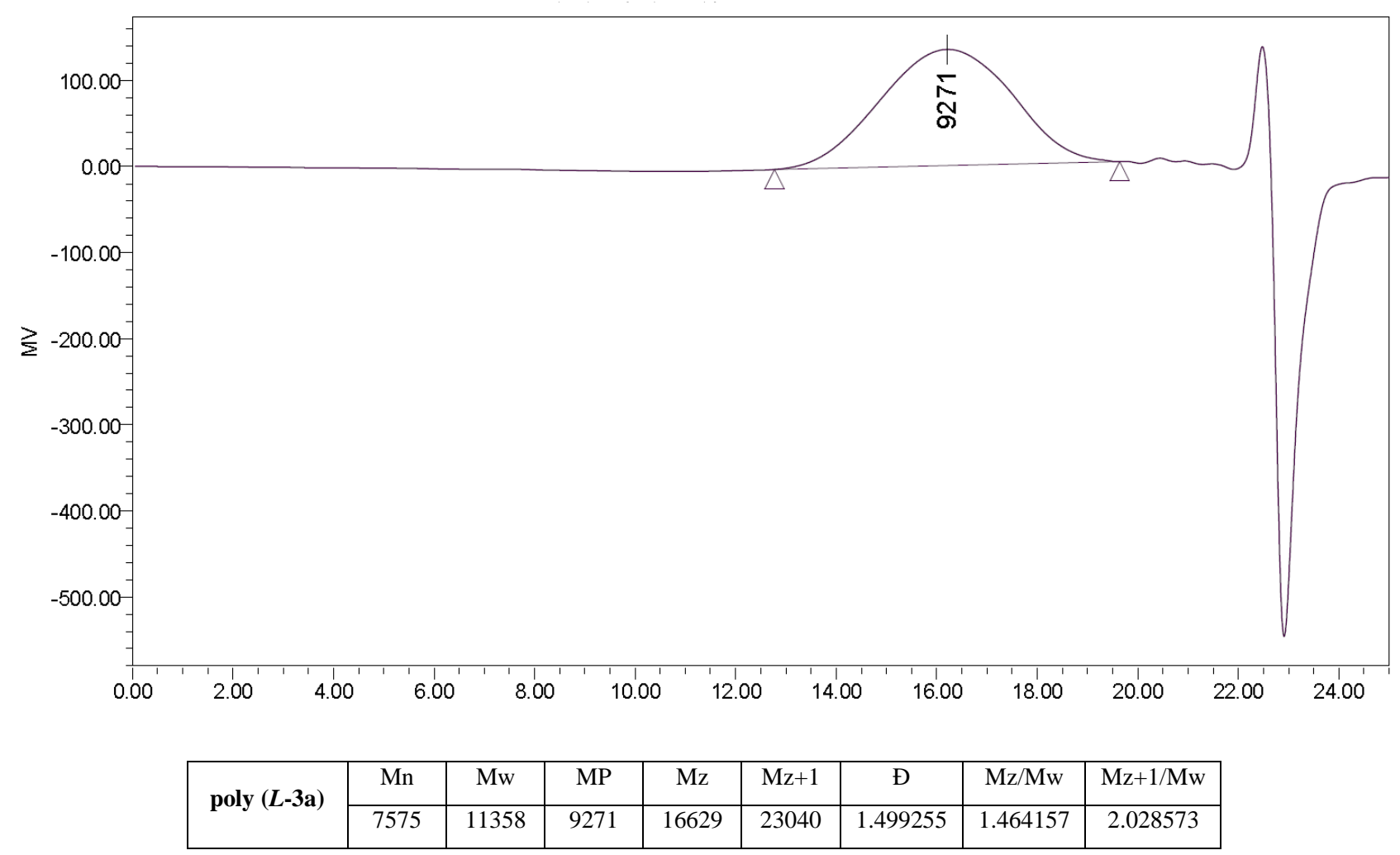

Figure S30. SEC data of Poly $(\boldsymbol{L}-\mathbf{3 a})$ 


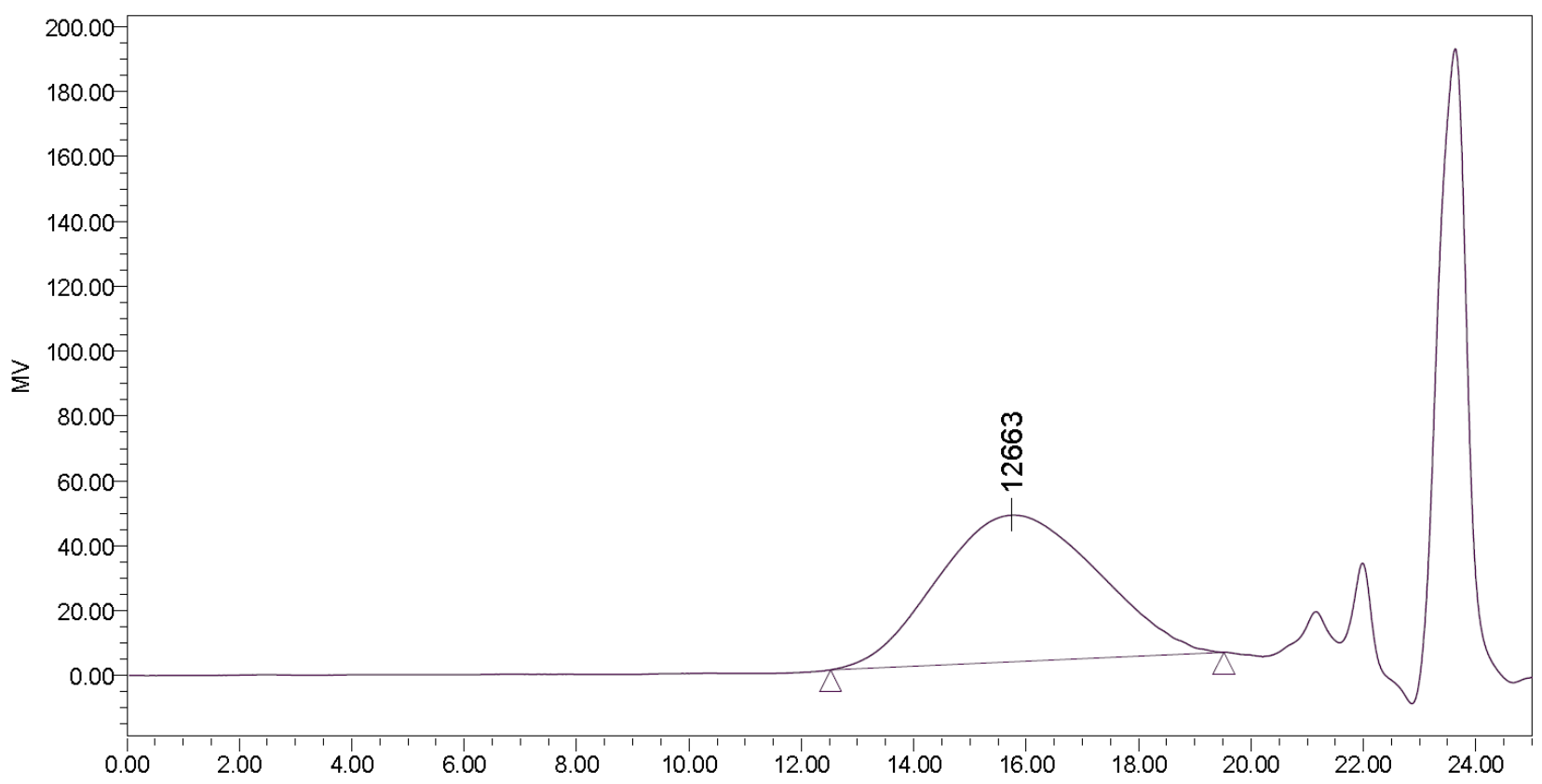

\begin{tabular}{|c|c|c|c|c|c|c|c|c|}
\hline \multirow{2}{*}{ poly $(\boldsymbol{L}$-9a) } & $\mathrm{Mn}$ & $\mathrm{Mw}$ & $\mathrm{MP}$ & $\mathrm{Mz}$ & $\mathrm{Mz}+1$ & Đ & $\mathrm{Mz} / \mathrm{Mw}$ & $\mathrm{Mz}+1 / \mathrm{Mw}$ \\
\cline { 2 - 9 } & 9087 & 14728 & 12663 & 22632 & 32002 & 1.620869 & 1.536641 & 2.172807 \\
\hline
\end{tabular}

Figure S31. SEC data of Poly $(\boldsymbol{L}-\mathbf{9 a})$

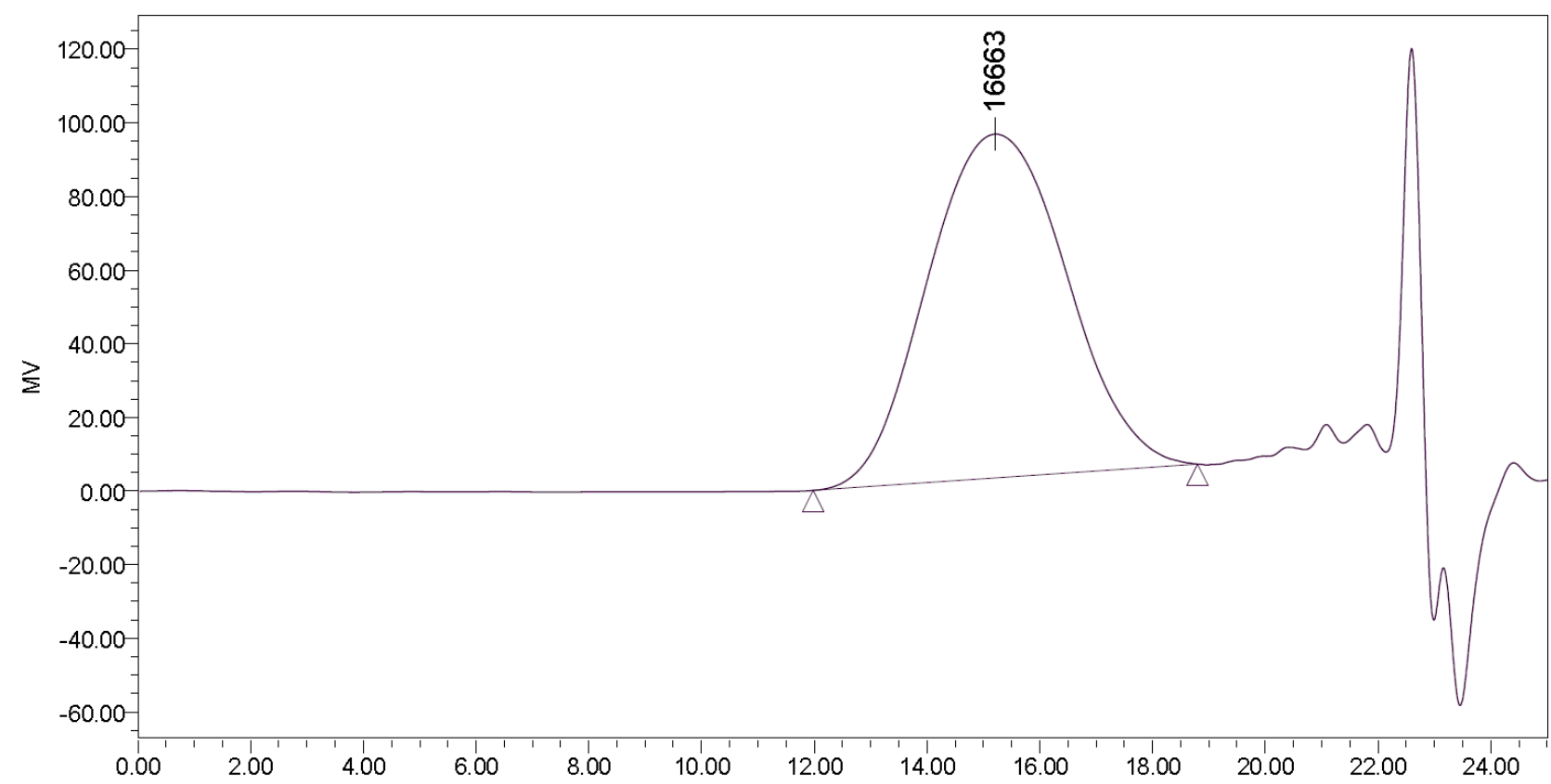

\begin{tabular}{|c|c|c|c|c|c|c|c|c|}
\hline \multirow{2}{*}{ poly (L-10a) } & $\mathrm{Mn}$ & $\mathrm{Mw}$ & $\mathrm{MP}$ & $\mathrm{Mz}$ & $\mathrm{Mz}+1$ & Đ & $\mathrm{Mz} / \mathrm{Mw}$ & $\mathrm{Mz}+1 / \mathrm{Mw}$ \\
\cline { 2 - 9 } & 13416 & 19334 & 16663 & 27393 & 37314 & 1.441167 & 1.416824 & 1.929938 \\
\hline
\end{tabular}

Figure S32. SEC data of Poly $(L-10 a)$ 


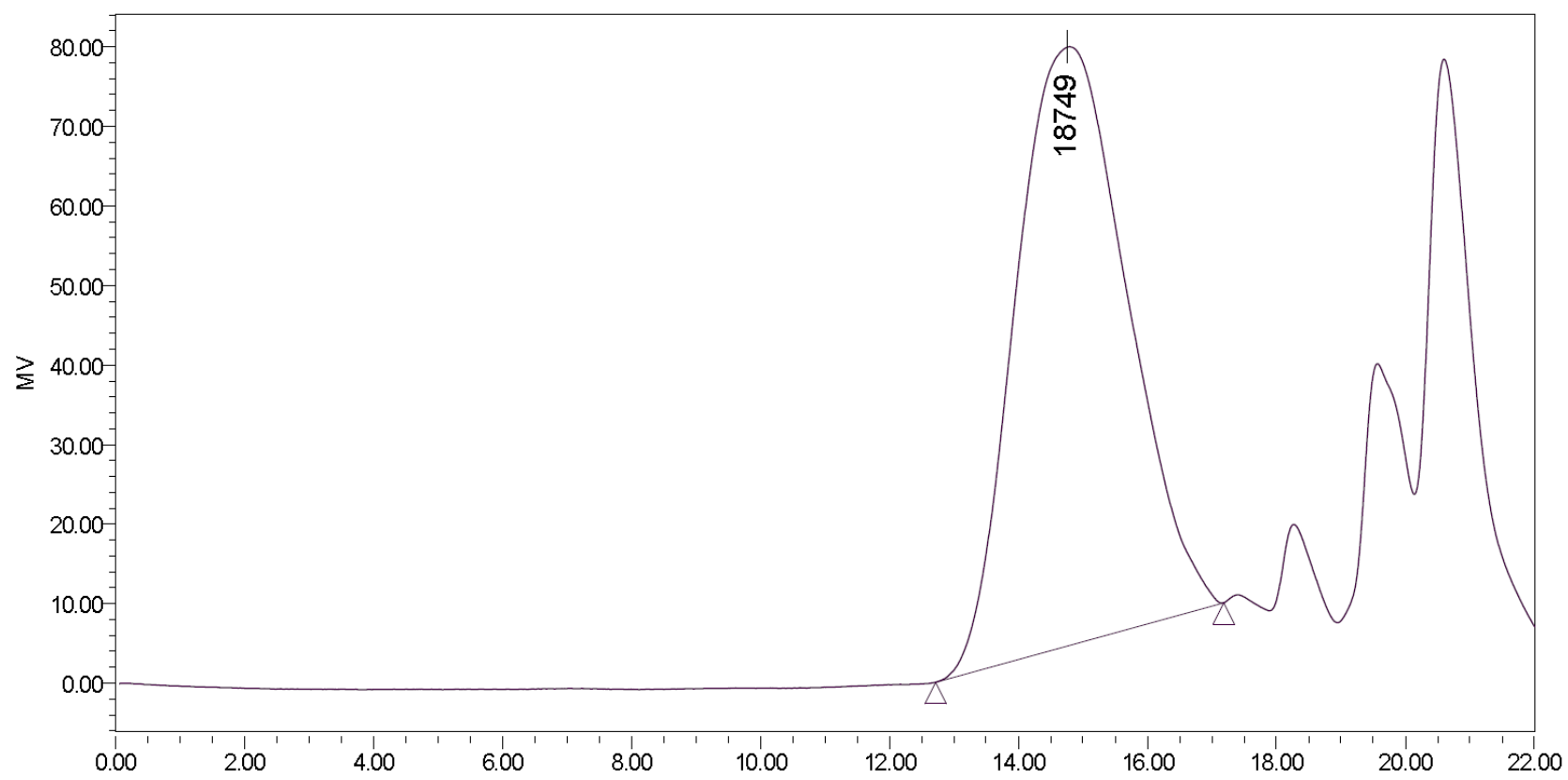

\begin{tabular}{|c|c|c|c|c|c|c|c|c|}
\hline \multirow{2}{*}{ poly $(\boldsymbol{D}$-9a) } & $\mathrm{Mn}$ & $\mathrm{Mw}$ & $\mathrm{MP}$ & $\mathrm{Mz}$ & $\mathrm{Mz}+1$ & Đ & $\mathrm{Mz} / \mathrm{Mw}$ & $\mathrm{Mz}+1 / \mathrm{Mw}$ \\
\cline { 2 - 9 } & 10999 & 23083 & 18749 & 40020 & 58014 & 2.098629 & 1.733776 & 2.51334 \\
\hline
\end{tabular}

Figure S33. SEC data of Poly (D-9a) 